\title{
Study of $B^{0} \rightarrow \rho^{+} \rho^{-}$decays and constraints on the CKM angle $\alpha$
}

B. Aubert, ${ }^{1}$ M. Bona, ${ }^{1}$ D. Boutigny, ${ }^{1}$ Y. Karyotakis, ${ }^{1}$ J. P. Lees, ${ }^{1}$ V. Poireau, ${ }^{1}$ X. Prudent,${ }^{1}$ V. Tisserand,${ }^{1}$ A. Zghiche, ${ }^{1}$ J. Garra Tico, ${ }^{2}$ E. Grauges, ${ }^{2}$ L. Lopez, ${ }^{3}$ A. Palano, ${ }^{3}$ G. Eigen, ${ }^{4}$ I. Ofte, ${ }^{4}$ B. Stugu, ${ }^{4}$ L. Sun, ${ }^{4}$ G. S. Abrams, ${ }^{5}$ M. Battaglia, D. N. Brown, ${ }^{5}$ J. Button-Shafer, ${ }^{5}$ R. N. Cahn, ${ }^{5}$ Y. Groysman, ${ }^{5}$ R. G. Jacobsen, ${ }^{5}$ J. A. Kadyk, ${ }^{5}$ L. T. Kerth, ${ }^{5}$ Yu. G. Kolomensky, ${ }^{5}$ G. Kukartsev, ${ }^{5}$ D. Lopes Pegna,${ }^{5}$ G. Lynch, ${ }^{5}$ L. M. Mir, ${ }^{5}$ T. J. Orimoto, ${ }^{5}$ M. Pripstein, ${ }^{5}$ N. A. Roe, ${ }^{5}$ M. T. Ronan, ${ }^{5, *}$ K. Tackmann, ${ }^{5}$ W. A. Wenzel, ${ }^{5}$ P. del Amo Sanchez, ${ }^{6}$ C. M. Hawkes, ${ }^{6}$ A. T. Watson, ${ }^{6}$ T. Held, ${ }^{7}$ H. Koch, ${ }^{7}$ B. Lewandowski, ${ }^{7}$ M. Pelizaeus, ${ }^{7}$ T. Schroeder, M. Steinke, ${ }^{7}$ W. N. Cottingham, ${ }^{8}$ D. Walker, ${ }^{8}$ D. J. Asgeirsson, ${ }^{9}$ T. Cuhadar-Donszelmann, ${ }^{9}$ B. G. Fulsom, ${ }^{9}$ C. Hearty, ${ }^{9}$ N. S. Knecht, ${ }^{9}$ T. S. Mattison, ${ }^{9}$ J. A. McKenna, ${ }^{9}$ A. Khan, ${ }^{10}$ M. Saleem, ${ }^{10}$ L. Teodorescu, ${ }^{10}$ V. E. Blinov, ${ }^{11}$ A. D. Bukin, ${ }^{11}$ V. P. Druzhinin, ${ }^{11}$ V. B. Golubev, ${ }^{11}$ A. P. Onuchin, ${ }^{11}$ S. I. Serednyakov, ${ }^{11}$ Yu. I. Skovpen, ${ }^{11}$ E. P. Solodov, ${ }^{11}$ K. Yu Todyshev,${ }^{11}$ M. Bondioli, ${ }^{12}$ S. Curry,${ }^{12}$ I. Eschrich, ${ }^{12}$ D. Kirkby, ${ }^{12}$ A. J. Lankford, ${ }^{12}$ P. Lund,${ }^{12}$ M. Mandelkern, ${ }^{12}$ E. C. Martin, ${ }^{12}$ D. P. Stoker ${ }^{12}$ S. Abachi, ${ }^{13}$ C. Buchanan, ${ }^{13}$ S. D. Foulkes, ${ }^{14}$ J. W. Gary, ${ }^{14}$ F. Liu, ${ }^{14}$ O. Long, ${ }^{14}$ B. C. Shen,${ }^{14}$ L. Zhang, ${ }^{14}$ H. P. Paar, ${ }^{15}$ S. Rahatlou, ${ }^{15}$ V. Sharma, ${ }^{15}$

J. W. Berryhill, ${ }^{16}$ C. Campagnari, ${ }^{16}$ A. Cunha, ${ }^{16}$ B. Dahmes,${ }^{16}$ T. M. Hong, ${ }^{16}$ D. Kovalskyi, ${ }^{16}$ J. D. Richman, ${ }^{16}$

T. W. Beck, ${ }^{17}$ A. M. Eisner, ${ }^{17}$ C. J. Flacco, ${ }^{17}$ C. A. Heusch, ${ }^{17}$ J. Kroseberg,,${ }^{17}$ W. S. Lockman, ${ }^{17}$ T. Schalk,,${ }^{17}$

B. A. Schumm, ${ }^{17}$ A. Seiden, ${ }^{17}$ D. C. Williams,${ }^{17}$ M. G. Wilson, ${ }^{17}$ L. O. Winstrom, ${ }^{17}$ E. Chen, ${ }^{18}$ C. H. Cheng, ${ }^{18}$ A. Dvoretskii, ${ }^{18}$ F. Fang, ${ }^{18}$ D. G. Hitlin, ${ }^{18}$ I. Narsky, ${ }^{18}$ T. Piatenko, ${ }^{18}$ F. C. Porter, ${ }^{18}$ G. Mancinelli, ${ }^{19}$ B. T. Meadows, ${ }^{19}$ K. Mishra, ${ }^{19}$ M. D. Sokoloff, ${ }^{19}$ F. Blanc,${ }^{20}$ P. C. Bloom,${ }^{20}$ S. Chen, ${ }^{20}$ W. T. Ford,${ }^{20}$ J. F. Hirschauer, ${ }^{20}$ A. Kreisel, ${ }^{20}$ M. Nagel, ${ }^{20}$ U. Nauenberg, ${ }^{20}$ A. Olivas, ${ }^{20}$ J. G. Smith,${ }^{20}$ K. A. Ulmer, ${ }^{20}$ S. R. Wagner, ${ }^{20}$ J. Zhang, ${ }^{20}$ A. M. Gabareen, ${ }^{21}$ A. Soffer, ${ }^{21}$ W. H. Toki, ${ }^{21}$ R. J. Wilson, ${ }^{21}$ F. Winklmeier, ${ }^{21}$ Q. Zeng, ${ }^{21}$ D. D. Altenburg, ${ }^{22}$ E. Feltresi, ${ }^{22}$ A. Hauke, ${ }^{22}$ H. Jasper, ${ }^{22}$ J. Merkel, ${ }^{22}$ A. Petzold, ${ }^{22}$ B. Spaan, ${ }^{22}$ K. Wacker, ${ }^{22}$ T. Brandt, ${ }^{23}$ V. Klose, ${ }^{23}$ H. M. Lacker, ${ }^{23}$ W. F. Mader,${ }^{23}$ R. Nogowski, ${ }^{23}$ J. Schubert, ${ }^{23}$ K. R. Schubert, ${ }^{23}$ R. Schwierz, ${ }^{23}$ J. E. Sundermann, ${ }^{23}$ A. Volk, ${ }^{23}$ D. Bernard, ${ }^{24}$

G. R. Bonneaud ${ }^{24}$ E. Latour, ${ }^{24}$ V. Lombardo,${ }^{24}$ Ch. Thiebaux,${ }^{24}$ M. Verderi, ${ }^{24}$ P. J. Clark, ${ }^{25}$ W. Gradl,${ }^{25}$ F. Muheim, ${ }^{25}$ S. Playfer, ${ }^{25}$ A. I. Robertson, ${ }^{25}$ Y. Xie, ${ }^{25}$ M. Andreotti, ${ }^{26}$ D. Bettoni,${ }^{26}$ C. Bozzi,${ }^{26}$ R. Calabrese, ${ }^{26}$ A. Cecchi, ${ }^{26}$ G. Cibinetto, ${ }^{26}$ P. Franchini, ${ }^{26}$ E. Luppi, ${ }^{26}$ M. Negrini, ${ }^{26}$ A. Petrella, ${ }^{26}$ L. Piemontese, ${ }^{26}$ E. Prencipe, ${ }^{26}$ V. Santoro, ${ }^{26}$

F. Anulli, ${ }^{27}$ R. Baldini-Ferroli, ${ }^{27}$ A. Calcaterra, ${ }^{27}$ R. de Sangro, ${ }^{27}$ G. Finocchiaro, ${ }^{27}$ S. Pacetti, ${ }^{27}$ P. Patteri, ${ }^{27}$ I. M. Peruzzi, ${ }^{27, \dagger}$ M. Piccolo, ${ }^{27}$ M. Rama, ${ }^{27}$ A. Zallo, ${ }^{27}$ A. Buzzo, ${ }^{28}$ R. Contri, ${ }^{28}$ M. Lo Vetere, ${ }^{28}$ M. M. Macri, ${ }^{28}$ M. R. Monge, ${ }^{28}$ S. Passaggio, ${ }^{28}$ C. Patrignani, ${ }^{28}$ E. Robutti, ${ }^{28}$ A. Santroni, ${ }^{28}$ S. Tosi, ${ }^{28}$ K. S. Chaisanguanthum, ${ }^{29}$ M. Morii, ${ }^{29}$ J. Wu, ${ }^{29}$ R. S. Dubitzky, ${ }^{30}$ J. Marks, ${ }^{30}$ S. Schenk ${ }^{30}$ U. Uwer,${ }^{30}$ D. J. Bard, ${ }^{31}$ P. D. Dauncey, ${ }^{31}$ R. L. Flack, ${ }^{31}$

J. A. Nash, ${ }^{31}$ M. B. Nikolich,${ }^{31}$ W. Panduro Vazquez, ${ }^{31}$ P. K. Behera, ${ }^{32}$ X. Chai, ${ }^{32}$ M. J. Charles,${ }^{32}$ U. Mallik, ${ }^{32}$

N. T. Meyer, ${ }^{32}$ V. Ziegler, ${ }^{32}$ J. Cochran, ${ }^{33}$ H. B. Crawley, ${ }^{33}$ L. Dong, ${ }^{33}$ V. Eyges, ${ }^{33}$ W. T. Meyer, ${ }^{33}$ S. Prell, ${ }^{33}$

E. I. Rosenberg, ${ }^{33}$ A. E. Rubin, ${ }^{33}$ A. V. Gritsan, ${ }^{34}$ Z. J. Guo, ${ }^{34}$ C. K. Lae, ${ }^{34}$ A. G. Denig, ${ }^{35}$ M. Fritsch, ${ }^{35}$ G. Schott, ${ }^{35}$ N. Arnaud, ${ }^{36}$ J. Béquilleux, ${ }^{36}$ M. Davier, ${ }^{36}$ G. Grosdidier, ${ }^{36}$ A. Höcker,${ }^{36}$ V. Lepeltier, ${ }^{36}$ F. Le Diberder, ${ }^{36}$ A. M. Lutz, ${ }^{36}$ S. Pruvot, ${ }^{36}$ S. Rodier, ${ }^{36}$ P. Roudeau, ${ }^{36}$ M. H. Schune, ${ }^{36}$ J. Serrano, ${ }^{36}$ V. Sordini, ${ }^{36}$ A. Stocchi, ${ }^{36}$ W. F. Wang, ${ }^{36}$ G. Wormser, ${ }^{36}$ D. J. Lange, ${ }^{37}$ D. M. Wright, ${ }^{37}$ C. A. Chavez, ${ }^{38}$ I. J. Forster, ${ }^{38}$ J. R. Fry, ${ }^{38}$ E. Gabathuler, ${ }^{38}$ R. Gamet, ${ }^{38}$ D. E. Hutchcroft, ${ }^{38}$ D. J. Payne, ${ }^{38}$ K. C. Schofield, ${ }^{38}$ C. Touramanis, ${ }^{38}$ A. J. Bevan, ${ }^{39}$ K. A. George,,${ }^{39}$ F. Di Lodovico, ${ }^{39}$

W. Menges, ${ }^{39}$ R. Sacco ${ }^{39}$ G. Cowan, ${ }^{40}$ H. U. Flaecher, ${ }^{40}$ D. A. Hopkins, ${ }^{40}$ P. S. Jackson, ${ }^{40}$ T. R. McMahon, ${ }^{40}$

F. Salvatore, ${ }^{40}$ A. C. Wren, ${ }^{40}$ D. N. Brown, ${ }^{41}$ C. L. Davis, ${ }^{41}$ J. Allison, ${ }^{42}$ N. R. Barlow, ${ }^{42}$ R. J. Barlow, ${ }^{42}$ Y. M. Chia, ${ }^{42}$ C. L. Edgar, ${ }^{42}$ G. D. Lafferty, ${ }^{42}$ T. J. West, ${ }^{42}$ J. I. Yi, ${ }^{42}$ J. Anderson, ${ }^{43}$ C. Chen,${ }^{43}$ A. Jawahery, ${ }^{43}$ D. A. Roberts, ${ }^{43}$ G. Simi, ${ }^{43}$ J. M. Tuggle, ${ }^{43}$ G. Blaylock,${ }^{44}$ C. Dallapiccola,${ }^{44}$ S. S. Hertzbach, ${ }^{44}$ X. Li,${ }^{44}$ T. B. Moore,${ }^{44}$ E. Salvati, ${ }^{44}$ S. Saremi, ${ }^{44}$ R. Cowan ${ }^{45}$ P. H. Fisher ${ }^{45}$ G. Sciolla, ${ }^{45}$ S. J. Sekula,${ }^{45}$ M. Spitznagel, ${ }^{45}$ F. Taylor, ${ }^{45}$ R. K. Yamamoto, ${ }^{45}$ S. E. Mclachlin, ${ }^{46}$ P. M. Patel, ${ }^{46}$ S. H. Robertson, ${ }^{46}$ A. Lazzaro, ${ }^{47}$ F. Palombo, ${ }^{47}$ J. M. Bauer, ${ }^{48}$ L. Cremaldi, ${ }^{48}$ V. Eschenburg, ${ }^{48}$ R. Godang, ${ }^{48}$

R. Kroeger, ${ }^{48}$ D. A. Sanders, ${ }^{48}$ D. J. Summers, ${ }^{48}$ H. W. Zhao, ${ }^{48}$ S. Brunet,${ }^{49}$ D. Côté, ${ }^{49}$ M. Simard,${ }^{49}$ P. Taras, ${ }^{49}$ F. B. Viaud, ${ }^{49}$ H. Nicholson, ${ }^{50}$ G. De Nardo, ${ }^{51}$ F. Fabozzi,${ }^{51, \$}$ L. Lista ${ }^{51}$ D. Monorchio,${ }^{51}$ C. Sciacca,${ }^{51}$ M. A. Baak, ${ }^{52}$ G. Raven, ${ }^{52}$ H. L. Snoek, ${ }^{52}$ C. P. Jessop, ${ }^{53}$ J. M. LoSecco, ${ }^{53}$ G. Benelli, ${ }^{54}$ L. A. Corwin, ${ }^{54}$ K. K. Gan, ${ }^{54}$ K. Honscheid, ${ }^{54}$ D. Hufnagel, ${ }^{54}$ H. Kagan, ${ }^{54}$ R. Kass, ${ }^{54}$ J. P. Morris,${ }^{54}$ A. M. Rahimi,${ }^{54}$ J. J. Regensburger,${ }^{54}$ R. Ter-Antonyan, ${ }^{54}$ Q. K. Wong, ${ }^{54}$ N. L. Blount, ${ }^{55}$ J. Brau, ${ }^{55}$ R. Frey, ${ }^{55}$ O. Igonkina, ${ }^{55}$ J. A. Kolb,${ }^{55}$ M. Lu, ${ }^{55}$ R. Rahmat,${ }^{55}$ N. B. Sinev, ${ }^{55}$

D. Strom, ${ }^{55}$ J. Strube,${ }^{55}$ E. Torrence, ${ }^{55}$ N. Gagliardi,${ }^{56}$ A. Gaz, ${ }^{56}$ M. Margoni,${ }^{56}$ M. Morandin,${ }^{56}$ A. Pompili, ${ }^{56}$

M. Posocco, ${ }^{56}$ M. Rotondo, ${ }^{56}$ F. Simonetto, ${ }^{56}$ R. Stroili, ${ }^{56}$ C. Voci, ${ }^{56}$ E. Ben-Haim, ${ }^{57}$ H. Briand, ${ }^{57}$ J. Chauveau, ${ }^{57}$ P. David ${ }^{57}$ L. Del Buono,${ }^{57}$ Ch. de la Vaissière, ${ }^{57}$ O. Hamon, ${ }^{57}$ B. L. Hartfiel, ${ }^{57}$ Ph. Leruste, ${ }^{57}$ J. Malclès,${ }^{57}$ J. Ocariz,${ }^{57}$ A. Perez,${ }^{57}$ L. Gladney, ${ }^{58}$ M. Biasini, ${ }^{59}$ R. Covarelli, ${ }^{59}$ E. Manoni, ${ }^{59}$ C. Angelini, ${ }^{60}$ G. Batignani, ${ }^{60}$ S. Bettarini, ${ }^{60}$ 
G. Calderini, ${ }^{60}$ M. Carpinelli, ${ }^{60}$ R. Cenci, ${ }^{60}$ A. Cervelli, ${ }^{60}$ F. Forti, ${ }^{60}$ M. A. Giorgi, ${ }^{60}$ A. Lusiani, ${ }^{60}$ G. Marchiori, ${ }^{60}$ M. A. Mazur, ${ }^{60}$ M. Morganti, ${ }^{60}$ N. Neri, ${ }^{60}$ E. Paoloni, ${ }^{60}$ G. Rizzo, ${ }^{60}$ J. J. Walsh, ${ }^{60}$ M. Haire, ${ }^{61}$ J. Biesiada, ${ }^{62}$ P. Elmer,${ }^{62}$ Y. P. Lau, ${ }^{62}$ C. Lu, ${ }^{62}$ J. Olsen, ${ }^{62}$ A. J. S. Smith ${ }^{62}$ A. V. Telnov,${ }^{62}$ E. Baracchini,${ }^{63}$ F. Bellini, ${ }^{63}$ G. Cavoto, ${ }^{63}$ A. D'Orazio, ${ }^{63}$

D. del Re ${ }^{63}$ E. Di Marco, ${ }^{63}$ R. Faccini ${ }^{63}$ F. Ferrarotto, ${ }^{63}$ F. Ferroni, ${ }^{63}$ M. Gaspero,${ }^{63}$ P. D. Jackson, ${ }^{63}$ L. Li Gioi, ${ }^{63}$ M. A. Mazzoni, ${ }^{63}$ S. Morganti, ${ }^{63}$ G. Piredda, ${ }^{63}$ F. Polci, ${ }^{63}$ F. Renga,${ }^{63}$ C. Voena,${ }^{63}$ M. Ebert, ${ }^{64}$ H. Schröder,${ }^{64}$ R. Waldi ${ }^{64}$ T. Adye,${ }^{65}$ G. Castelli, ${ }^{65}$ B. Franek,${ }^{65}$ E. O. Olaiya, ${ }^{65}$ S. Ricciardi, ${ }^{65}$ W. Roethel,${ }^{65}$ F. F. Wilson, ${ }^{65}$ R. Aleksan,${ }^{66}$ S. Emery, ${ }^{66}$ M. Escalier, ${ }^{66}$ A. Gaidot, ${ }^{66}$ S. F. Ganzhur, ${ }^{66}$ P. F. Giraud ${ }^{66}$ G. Hamel de Monchenault, ${ }^{66}$ W. Kozanecki, ${ }^{66}$ M. Legendre, ${ }^{66}$ G. Vasseur, ${ }^{66}$ Ch. Yèche, ${ }^{66}$ M. Zito, ${ }^{66}$ X. R. Chen, ${ }^{67}$ H. Liu, ${ }^{67}$ W. Park, ${ }^{67}$ M. V. Purohit, ${ }^{67}$ J. R. Wilson, ${ }^{67}$ M. T. Allen, ${ }^{68}$ D. Aston, ${ }^{68}$ R. Bartoldus, ${ }^{68}$ P. Bechtle,${ }^{68}$ N. Berger,${ }^{68}$ R. Claus, ${ }^{68}$ J. P. Coleman, ${ }^{68}$ M. R. Convery, ${ }^{68}$ J. C. Dingfelder ${ }^{68}$ J. Dorfan, ${ }^{68}$ G. P. Dubois-Felsmann, ${ }^{68}$ D. Dujmic, ${ }^{68}$ W. Dunwoodie, ${ }^{68}$ R. C. Field, ${ }^{68}$ T. Glanzman, ${ }^{68}$ S. J. Gowdy, ${ }^{68}$ M. T. Graham, ${ }^{68}$ P. Grenier, ${ }^{68}$ C. Hast, ${ }^{68}$ T. Hryn'ova, ${ }^{68}$ W. R. Innes,${ }^{68}$ M. H. Kelsey, ${ }^{68}$ H. Kim, ${ }^{68}$ P. Kim, ${ }^{68}$ D. W. G. S. Leith, ${ }^{68}$ S. Li, ${ }^{68}$ S. Luitz, ${ }^{68}$ V. Luth, ${ }^{68}$ H. L. Lynch, ${ }^{68}$ D. B. MacFarlane, ${ }^{68}$ H. Marsiske, ${ }^{68}$ R. Messner ${ }^{68}$ D. R. Muller, ${ }^{68}$ C. P. O' Grady, ${ }^{68}$ A. Perazzo, ${ }^{68}$ M. Perl, ${ }^{68}$ T. Pulliam, ${ }^{68}$ B. N. Ratcliff, ${ }^{68}$ A. Roodman, ${ }^{68}$ A. A. Salnikov, ${ }^{68}$ R. H. Schindler, ${ }^{68}$ J. Schwiening, ${ }^{68}$ A. Snyder, ${ }^{68}$ J. Stelzer ${ }^{68}$ D. Su, ${ }^{68}$ M. K. Sullivan, ${ }^{68}$ K. Suzuki, ${ }^{68}$ S. K. Swain, ${ }^{68}$ J. M. Thompson, ${ }^{68}$ J. Va'vra, ${ }^{68}$ N. van Bakel, ${ }^{68}$ A. P. Wagner, ${ }^{68}$ M. Weaver, ${ }^{68}$ W. J. Wisniewski, ${ }^{68} \mathrm{M}$. Wittgen, ${ }^{68}$ D. H. Wright, ${ }^{68}$ A. K. Yarritu, ${ }^{68}$ K. Yi, ${ }^{68}$ C. C. Young, ${ }^{68}$ P. R. Burchat,${ }^{69}$ A. J. Edwards, ${ }^{69}$ S. A. Majewski, ${ }^{69}$ B. A. Petersen, ${ }^{69}$ L. Wilden, ${ }^{69}$ S. Ahmed, ${ }^{70}$ M. S. Alam, ${ }^{70}$ R. Bula ${ }^{70}$ J. A. Ernst, ${ }^{70}$ V. Jain, ${ }^{70}$ B. Pan, ${ }^{70}$ M. A. Saeed, ${ }^{70}$ F. R. Wappler, ${ }^{70}$ S. B. Zain, ${ }^{70}$ W. Bugg, ${ }^{71}$ M. Krishnamurthy, ${ }^{71}$ S. M. Spanier, ${ }^{71}$ R. Eckmann, ${ }^{72}$ J. L. Ritchie, ${ }^{72}$ A. M. Ruland, ${ }^{72}$ C. J. Schilling, ${ }^{72}$ R. F. Schwitters, ${ }^{72}$ J. M. Izen, ${ }^{73}$ X. C. Lou, ${ }^{73}$ S. Ye, ${ }^{73}$ F. Bianchi,${ }^{74}$ F. Gallo, ${ }^{74}$ D. Gamba, ${ }^{74}$ M. Pelliccioni, ${ }^{74}$ M. Bomben, ${ }^{75}$ L. Bosisio, ${ }^{75}$ C. Cartaro, ${ }^{75}$ F. Cossutti, ${ }^{75}$ G. Della Ricca, ${ }^{75}$ L. Lanceri, ${ }^{75}$ L. Vitale, ${ }^{75}$ V. Azzolini, ${ }^{76}$ N. Lopez-March, ${ }^{76}$ F. Martinez-Vidal, ${ }^{76}$ D. A. Milanes, ${ }^{76}$ A. Oyanguren, ${ }^{76}$ J. Albert, ${ }^{77}$ Sw. Banerjee, ${ }^{77}$ B. Bhuyan, ${ }^{77}$ K. Hamano,${ }^{77}$ R. Kowalewski, ${ }^{77}$ I. M. Nugent, ${ }^{77}$ J. M. Roney, ${ }^{77}$ R. J. Sobie ${ }^{77}$ J. J. Back, ${ }^{78}$ P. F. Harrison, ${ }^{78}$ T. E. Latham, ${ }^{78}$ G. B. Mohanty, ${ }^{78}$ M. Pappagallo, ${ }^{78,8}$ H. R. Band, ${ }^{79}$ X. Chen,${ }^{79}$ S. Dasu, ${ }^{79}$ K. T. Flood, ${ }^{79}$ J. J. Hollar, ${ }^{79}$ P. E. Kutter, ${ }^{79}$ Y. Pan, ${ }^{79}$ M. Pierini, ${ }^{79}$ R. Prepost, ${ }^{79}$ S. L. Wu, ${ }^{79}$ Z. Yu, ${ }^{79}$ and H. Neal ${ }^{80}$

(BABAR Collaboration)

\footnotetext{
${ }^{1}$ Laboratoire de Physique des Particules, IN2P3/CNRS et Université de Savoie, F-74941 Annecy-Le-Vieux, France

${ }^{2}$ Universitat de Barcelona, Facultat de Fisica, Departament ECM, E-08028 Barcelona, Spain

${ }^{3}$ Università di Bari, Dipartimento di Fisica and INFN, I-70126 Bari, Italy

${ }^{4}$ University of Bergen, Institute of Physics, N-5007 Bergen, Norway

${ }^{5}$ Lawrence Berkeley National Laboratory and University of California, Berkeley, California 94720, USA

${ }^{6}$ University of Birmingham, Birmingham, B15 2TT, United Kingdom

${ }^{7}$ Ruhr Universität Bochum, Institut für Experimentalphysik 1, D-44780 Bochum, Germany

${ }^{8}$ University of Bristol, Bristol BS8 1TL, United Kingdom

${ }^{9}$ University of British Columbia, Vancouver, British Columbia, Canada V6T 1Z1

${ }^{10}$ Brunel University, Uxbridge, Middlesex UB8 3PH, United Kingdom

${ }^{11}$ Budker Institute of Nuclear Physics, Novosibirsk 630090, Russia

${ }^{12}$ University of California at Irvine, Irvine, California 92697, USA

${ }^{13}$ University of California at Los Angeles, Los Angeles, California 90024, USA

${ }^{14}$ University of California at Riverside, Riverside, California 92521, USA

${ }^{15}$ University of California at San Diego, La Jolla, California 92093, USA

${ }^{16}$ University of California at Santa Barbara, Santa Barbara, California 93106, USA

${ }^{17}$ University of California at Santa Cruz, Institute for Particle Physics, Santa Cruz, California 95064, USA

${ }^{18}$ California Institute of Technology, Pasadena, California 91125, USA

${ }^{19}$ University of Cincinnati, Cincinnati, Ohio 45221, USA

${ }^{20}$ University of Colorado, Boulder, Colorado 80309, USA

${ }^{21}$ Colorado State University, Fort Collins, Colorado 80523, USA

${ }^{22}$ Universität Dortmund, Institut für Physik, D-44221 Dortmund, Germany

${ }^{23}$ Technische Universität Dresden, Institut für Kern- und Teilchenphysik, D-01062 Dresden, Germany

${ }^{24}$ Laboratoire Leprince-Ringuet, CNRS/IN2P3, Ecole Polytechnique, F-91128 Palaiseau, France

${ }^{25}$ University of Edinburgh, Edinburgh EH9 3JZ, United Kingdom

${ }^{26}$ Università di Ferrara, Dipartimento di Fisica and INFN, I-44100 Ferrara, Italy

${ }^{27}$ Laboratori Nazionali di Frascati dell'INFN, I-00044 Frascati, Italy

${ }^{28}$ Università di Genova, Dipartimento di Fisica and INFN, I-16146 Genova, Italy

${ }^{29}$ Harvard University, Cambridge, Massachusetts 02138, USA
} 
${ }^{30}$ Universität Heidelberg, Physikalisches Institut, Philosophenweg 12, D-69120 Heidelberg, Germany

${ }^{31}$ Imperial College London, London, SW7 2AZ, United Kingdom

${ }^{32}$ University of Iowa, Iowa City, Iowa 52242, USA

${ }^{33}$ Iowa State University, Ames, Iowa 50011-3160, USA

${ }^{34}$ Johns Hopkins University, Baltimore, Maryland 21218, USA

${ }^{35}$ Universität Karlsruhe, Institut für Experimentelle Kernphysik, D-76021 Karlsruhe, Germany

${ }^{36}$ Laboratoire de l'Accélérateur Linéaire, IN2P3/CNRS et Université Paris-Sud 11,

Centre Scientifique d'Orsay, B. P. 34, F-91898 ORSAY Cedex, France

${ }^{37}$ Lawrence Livermore National Laboratory, Livermore, California 94550, USA

${ }^{38}$ University of Liverpool, Liverpool L69 7ZE, United Kingdom

${ }^{39}$ Queen Mary, University of London, E1 4NS, United Kingdom

${ }^{40}$ University of London, Royal Holloway and Bedford New College, Egham, Surrey TW20 OEX, United Kingdom

${ }^{41}$ University of Louisville, Louisville, Kentucky 40292, USA

${ }^{42}$ University of Manchester, Manchester M13 9PL, United Kingdom

${ }^{43}$ University of Maryland, College Park, Maryland 20742, USA

${ }^{44}$ University of Massachusetts, Amherst, Massachusetts 01003, USA

${ }^{45}$ Massachusetts Institute of Technology, Laboratory for Nuclear Science, Cambridge, Massachusetts 02139, USA

${ }^{46}$ Mc Gill University, Montréal, Québec, Canada H3A $2 T 8$

${ }^{47}$ Università di Milano, Dipartimento di Fisica and INFN, I-20133 Milano, Italy

${ }^{48}$ University of Mississippi, University, Mississippi 38677, USA

${ }^{49}$ Université de Montréal, Physique des Particules, Montréal, Québec, Canada H3C 3J7

${ }^{50}$ Mount Holyoke College, South Hadley, Massachusetts 01075, USA

${ }^{51}$ Università di Napoli Federico II, Dipartimento di Scienze Fisiche and INFN, I-80126, Napoli, Italy

${ }^{52}$ NIKHEF, National Institute for Nuclear Physics and High Energy Physics, NL-1009 DB Amsterdam, The Netherlands

${ }^{53}$ University of Notre Dame, Notre Dame, Indiana 46556, USA

${ }^{54}$ Ohio State University, Columbus, Ohio 43210, USA

${ }^{55}$ University of Oregon, Eugene, Oregon 97403, USA

${ }^{56}$ Università di Padova, Dipartimento di Fisica and INFN, I-35131 Padova, Italy

${ }^{57}$ Laboratoire de Physique Nucléaire et de Hautes Energies, IN2P3/CNRS, Université Pierre et Marie Curie-Paris6, Université Denis Diderot-Paris7, F-75252 Paris, France

${ }^{58}$ University of Pennsylvania, Philadelphia, Pennsylvania 19104, USA

${ }^{59}$ Università di Perugia, Dipartimento di Fisica and INFN, I-06100 Perugia, Italy

${ }^{60}$ Università di Pisa, Dipartimento di Fisica, Scuola Normale Superiore and INFN, I-56127 Pisa, Italy

${ }^{61}$ Prairie View A\&M University, Prairie View, Texas 77446, USA

${ }^{62}$ Princeton University, Princeton, New Jersey 08544, USA

${ }^{63}$ Università di Roma La Sapienza, Dipartimento di Fisica and INFN, I-00185 Roma, Italy

${ }^{64}$ Universität Rostock, D-18051 Rostock, Germany

${ }^{65}$ Rutherford Appleton Laboratory, Chilton, Didcot, Oxon, OX11 0QX, United Kingdom

${ }^{66}$ DSM/Dapnia, CEA/Saclay, F-91191 Gif-sur-Yvette, France

${ }^{67}$ University of South Carolina, Columbia, South Carolina 29208, USA

${ }^{68}$ Stanford Linear Accelerator Center, Stanford, California 94309, USA

${ }^{69}$ Stanford University, Stanford, California 94305-4060, USA

${ }^{70}$ State University of New York, Albany, New York 12222, USA

${ }^{71}$ University of Tennessee, Knoxville, Tennessee 37996, USA

${ }^{72}$ University of Texas at Austin, Austin, Texas 78712, USA

${ }^{73}$ University of Texas at Dallas, Richardson, Texas 75083, USA

${ }^{74}$ Università di Torino, Dipartimento di Fisica Sperimentale and INFN, I-10125 Torino, Italy

${ }^{75}$ Università di Trieste, Dipartimento di Fisica and INFN, I-34127 Trieste, Italy

${ }^{76}$ IFIC, Universitat de Valencia-CSIC, E-46071 Valencia, Spain

${ }^{77}$ University of Victoria, Victoria, British Columbia, Canada V8W $3 P 6$

${ }^{78}$ Department of Physics, University of Warwick, Coventry CV4 7AL, United Kingdom

${ }^{79}$ University of Wisconsin, Madison, Wisconsin 53706, USA

${ }^{80}$ Yale University, New Haven, Connecticut 06511, USA

(Received 15 May 2007; published 28 September 2007)

\footnotetext{
*Deceased.

†Also with Università di Perugia, Dipartimento di Fisica, Perugia, Italy.

*Also with Università della Basilicata, Potenza, Italy.

${ }^{\S}$ Also with IPPP, Physics Department, Durham University, Durham DH1 3LE, United Kingdom.
} 
We present results from an analysis of $B^{0} \rightarrow \rho^{+} \rho^{-}$decays using $(383.6 \pm 4.2) \times 10^{6} B \bar{B}$ pairs collected by the $B A B A R$ detector at the PEP-II asymmetric-energy $B$ factory at SLAC. The measurements of the $B^{0} \rightarrow \rho^{+} \rho^{-}$branching fraction, longitudinal polarization fraction $f_{L}$, and the $C P$-violating parameters $S_{\text {long }}$ and $C_{\text {long }}$ are as follows: $\mathcal{B}\left(B^{0} \rightarrow \rho^{+} \rho^{-}\right)=\left(25.5 \pm 2.1\right.$ (stat) ${ }_{-3.9}^{+3.6}($ syst $\left.)\right) \times 10^{-6}, f_{L}=$ $0.992 \pm 0.024(\text { stat })_{-0.013}^{+0.026}$ (syst),$\quad S_{\text {long }}=-0.17 \pm 0.20$ (stat) ${ }_{-0.06}^{+0.05}$ (syst), $\quad C_{\text {long }}=0.01 \pm 0.15$ (stat) \pm 0.06 (syst). We determine the unitarity triangle angle $\alpha$, using an isospin analysis of $B \rightarrow \rho \rho$ decays. One of the two solutions, $\alpha=[73.1,117.0]^{\circ}$ at $68 \%$ confidence level, is compatible with standard modelbased fits of existing data. Constraints on the unitarity triangle are also evaluated using an $S U(3)$ symmetry-based approach.

\section{INTRODUCTION}

Charge conjugation-parity $(C P)$ violation was first seen in the decay of neutral kaons [1]. It was shown some 40 years ago that $C P$ violation is a necessary but insufficient condition required to generate a net baryon-antibaryon asymmetry in the Universe [2]. The standard model (SM) of electroweak interactions describes $C P$ violation as a consequence of a complex phase in the three-generation Cabibbo-Kobayashi-Maskawa (CKM) quark-mixing matrix $[3,4]$ :

$$
V_{\mathrm{CKM}}=\left(\begin{array}{ccc}
V_{\mathrm{ud}} & V_{\mathrm{us}} & V_{\mathrm{ub}} \\
V_{\mathrm{cd}} & V_{\mathrm{cs}} & V_{\mathrm{cb}} \\
V_{\mathrm{td}} & V_{\mathrm{ts}} & V_{\mathrm{tb}}
\end{array}\right) .
$$

$V_{\mathrm{CKM}}$ describes the couplings of the $u, c$, and $t$ quarks to $d$, $s$, and $b$ quarks, which are mediated by the exchange of a $W$ boson. In $B$-meson decays the $C P$ violating parameters of the SM are most directly related to the angles and sides of the so-called unitarity triangle (UT), shown in Fig. 1. The angles $\alpha, \beta$, and $\gamma$ are defined as

$$
\begin{gathered}
\alpha \equiv \arg \left[-V_{\mathrm{td}} V_{\mathrm{tb}}^{*} / V_{\mathrm{ud}} V_{\mathrm{ub}}^{*}\right], \\
\beta \equiv \arg \left[-V_{\mathrm{cd}} V_{\mathrm{cb}}^{*} / V_{\mathrm{td}} V_{\mathrm{tb}}^{*}\right], \\
\gamma \equiv \arg \left[-V_{\mathrm{ud}} V_{\mathrm{ub}}^{*} / V_{\mathrm{cd}} V_{\mathrm{cb}}^{*}\right] .
\end{gathered}
$$

Any nontrivial phase in $V_{\mathrm{ij}}$ is $C P$ violating. $C P$ violating phases originating from the CKM matrix are related to weak interactions, and therefore referred to as weak phases. In the Wolfenstein convention [5], the angle $\gamma$ is the phase of $V_{\mathrm{ub}}, \beta$ is the phase of $V_{\mathrm{td}}$, and $\alpha$ is the phase difference between $V_{\mathrm{ub}}$ and $V_{\mathrm{td}}$ constrained to satisfy $\alpha=$ $\pi-\beta-\gamma$ through the unitarity of $V_{\mathrm{CKM}}$.

In Fig. 1 the UT is shown in the complex $(\bar{\rho}, \bar{\eta})$ plane, where the apex is given by the phase-convention indepen-

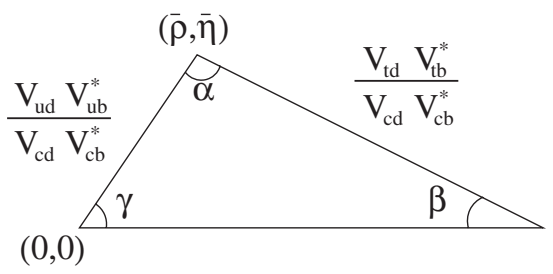

FIG. 1. The unitarity triangle in the $\bar{\rho}-\bar{\eta}$ plane. dent definition [6]:

$$
\bar{\rho}+i \bar{\eta} \equiv-\frac{V_{\mathrm{ud}} V_{\mathrm{ub}}^{*}}{V_{\mathrm{cd}} V_{\mathrm{cb}}^{*}}
$$

The quest to understand $C P$ violation remains, despite its observation in the $B$ meson system by both the $B A B A R$ [7] and Belle experiments [8], since the SM does not, through the CKM phase, incorporate enough $C P$ violation to explain the current matter-antimatter asymmetry $[9,10]$. The CKM angle $\beta$ is measured to a $1^{\circ}$ precision with $b \rightarrow$ $c \bar{c} s$ decays $[11,12]$ and is consistent with current predictions [13-15]. A significant deviation from results of SMbased fits of existing data for any of the UT angles would be a clear indication of new physics [16,17].

In principle, direct experimental measurements of $\alpha$ can be made from decays that proceed mainly through a $\bar{b} \rightarrow$ $u \bar{u} d$ tree diagram such as $B^{0} \rightarrow \rho^{+} \rho^{-}, \rho^{ \pm} \pi^{\overline{ }}, \pi^{+} \pi^{-}$, and $a_{1} \pi[18,19]$. Interference between the direct decay and decay after $B^{0} \bar{B}^{0}$ mixing in each of these decays results in a time-dependent decay-rate asymmetry that is sensitive to the angle $\alpha$. Figure 2 shows the leading order tree and gluonic penguin loop contributions to the decay $B^{0} \rightarrow$ $\rho^{+} \rho^{-}$. The presence of penguin contributions complicates the extraction of $\alpha$ from these decays. Using isospin relations [20], measurements of the $B^{+} \rightarrow \rho^{+} \rho^{0}$ [21,22] and $B^{0} \rightarrow \rho^{0} \rho^{0}[23]$ branching fractions show that the penguin contribution in $B \rightarrow \rho \rho$ is smaller than the leading tree diagram. The use of $S U(3)$ flavor symmetry to increase the precision on the weak phase constrained using $B^{0} \rightarrow$ $\rho^{+} \rho^{-}$and $B^{+} \rightarrow K^{* 0} \rho^{+}$decays has also been proposed [24]. Both of these approaches are discussed in Sec. X.

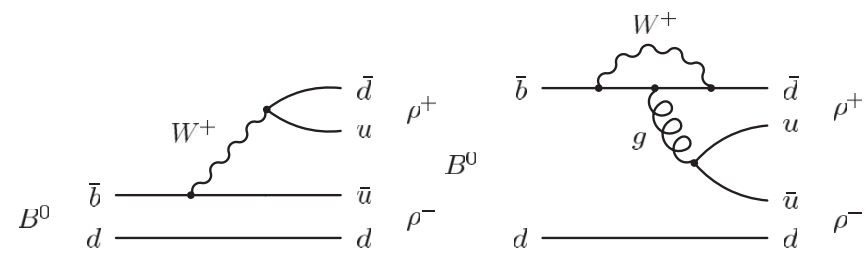

FIG. 2. Tree (top) and gluonic penguin (bottom) diagrams contributing to the process $B^{0} \rightarrow \rho^{+} \rho^{-}$. The penguin contribution coming from the diagram with a top quark in the loop dominates as contributions from processes with $u$ and $c$ quarks are suppressed. 
Section X and Ref. [25] describe a number of possible sources of theoretical uncertainty.

In $B^{0} \rightarrow \rho^{+} \rho^{-}$decays, a spin 0 particle (the $B^{0}$ meson) decays into two spin 1 particles ( $\rho^{ \pm}$mesons), as shown in Fig. 3. Subsequently, each $\rho^{ \pm}$meson decays into a $\pi^{ \pm} \pi^{0}$ pair. As a result, the $C P$ analysis of $B^{0} \rightarrow \rho^{+} \rho^{-}$is complicated by the presence of one amplitude with longitudinal polarization and two amplitudes with transverse polarization. The longitudinal amplitude is $C P$ even, while the transverse amplitudes contain $C P$-even and $C P$-odd states. The decay is observed to be dominated by the longitudinal polarization [26,27], with a fraction $f_{L}$ defined as the fraction of the helicity zero state in the decay. Integrating over the angle between the $\rho$ decay planes $\phi$, the angular decay rate is

$$
\begin{aligned}
\frac{d^{2} \Gamma}{\Gamma d \cos \theta_{1} d \cos \theta_{2}}= & \frac{9}{4}\left[f_{L} \cos ^{2} \theta_{1} \cos ^{2} \theta_{2}\right. \\
& \left.+\frac{1}{4}\left(1-f_{L}\right) \sin ^{2} \theta_{1} \sin ^{2} \theta_{2}\right],
\end{aligned}
$$

where the helicity angles $\theta_{i=1,2}$ are the angles between the $\pi^{0}$ momentum and the direction opposite to that of the $B^{0}$ in the $\rho$ rest frame.

We identify (tag) the initial flavor of the reconstructed $B$ candidate, $B_{\text {rec }}$, using information from the other $B$ meson, $B_{\text {tag }}$, in the event. The decay rate $f_{+}\left(f_{-}\right)$for a neutral $B$ meson decaying into a $C P$ eigenstate, where the $B_{\text {tag }}$ is a $B^{0}$ $\left(\bar{B}^{0}\right)$, is given by

$$
\begin{aligned}
f_{ \pm}(\Delta t)= & \frac{e^{-|\Delta t| / \tau_{B^{0}}}}{4 \tau_{B^{0}}}\left\{1 \pm \eta_{f}\left[S \sin \left(\Delta m_{d} \Delta t\right)\right.\right. \\
& \left.\left.-C \cos \left(\Delta m_{d} \Delta t\right)\right]\right\} .
\end{aligned}
$$

Here $\Delta t$ is the proper time difference between the decay of the $B_{\text {rec }}$ and $B_{\text {tag }}$ mesons in an event, $\tau_{B^{0}}$ is the mean $B^{0}$ lifetime, $\Delta m_{d}$ is the $B^{0}-\bar{B}^{0}$ mixing frequency [28], and $\eta_{f}$ is the $C P$ eigenvalue of the decay. This assumes that there is no difference between the $B^{0}$ and $\bar{B}^{0}$ lifetimes, $\Delta \Gamma=0$. The parameters $S$ and $C$ describe $B$-mixing induced and direct $C P$ violation, respectively. For the longitudinal polarization $\left(\eta_{f}=+1\right) S=S_{\text {long }}$ and $C=C_{\text {long }}$ are defined as

$$
\begin{gathered}
S_{\text {long }}=\frac{2 I m \lambda_{C P}}{1+\left|\lambda_{C P}\right|^{2}}, \\
C_{\text {long }}=\frac{1-\left|\lambda_{C P}\right|^{2}}{1+\left|\lambda_{C P}\right|^{2}},
\end{gathered}
$$

where $\lambda_{C P}=\frac{q}{p} \frac{\bar{A}}{A}$ [29], $q$ and $p$ are complex constants that relate the $B$ meson flavor eigenstates to the mass eigenstates, and $\bar{A} / A$ is the ratio of amplitudes of the decay of a $\bar{B}^{0}$ or $B^{0}$ to the final state under study. $C P$ violation is probed by studying the time-dependent decay-rate asymmetry

$$
\mathcal{A}(\Delta t)=\frac{\Gamma(\Delta t)-\bar{\Gamma}(\Delta t)}{\Gamma(\Delta t)+\bar{\Gamma}(\Delta t)},
$$

where $\Gamma(\bar{\Gamma})$ is the decay rate for $B^{0}\left(\bar{B}^{0}\right)$ tagged events. This asymmetry has the form

$$
\mathcal{A}(\Delta t)=S \sin \left(\Delta m_{d} \Delta t\right)-C \cos \left(\Delta m_{d} \Delta t\right) .
$$

The transverse polarization is an admixture of $C P$-even $\left(\eta_{f}=+1\right)$ and $C P$-odd $\left(\eta_{f}=-1\right)$ parts, where each $C P$ eigenstate has a decay-rate distribution of the form of Eq. (7). As the longitudinal polarization dominates this decay, we describe the $C P$ admixture of the transverse polarization with common parameters $S_{\text {tran }}$ and $C_{\text {tran }}$. We set $S_{\text {tran }}=C_{\text {tran }}=0$, and vary these parameters when evaluating systematic uncertainties.

In the absence of penguin contributions in $B^{0} \rightarrow \rho^{+} \rho^{-}$, $S_{\text {long }}=\sin 2 \alpha$ and $C_{\text {long }}=0$. The presence of penguin contributions with different weak phases to the tree-level amplitude shifts the experimentally measurable parameter $\alpha_{\text {eff }}$ away from the value of $\alpha$. In the presence of penguin contributions $\alpha_{\text {eff }}=\alpha+\delta \alpha$, where

$$
S_{\text {long }}=\sqrt{1-C_{\text {long }}^{2}} \sin 2 \alpha_{\text {eff }}
$$

and $C_{\text {long }}$ can be nonzero.

For $B^{+} \rightarrow \rho^{+} \rho^{0}$ one measures a time-integrated charge asymmetry, which is analogous to Eq. (10) and is defined as

TABLE I. Measurements of the $B \rightarrow \rho \rho$ decays. Branching fractions $(\mathcal{B})$ are in units of $10^{-6}$. The column marked $C_{\text {long }}\left(A_{C P}\right)$ denotes the time-dependent charge asymmetry $C_{\text {long }}$ in the case of the $B^{0} \rightarrow \rho^{+} \rho^{-}$decay, and the time-integrated charge asymmetry $A_{C P}$ in the case of $B^{ \pm} \rightarrow \rho^{ \pm} \rho^{0}$.

\begin{tabular}{l|ccccc}
\hline \hline Mode & Experiment (luminosity fb $\left.\mathrm{fb}^{-1}\right)$ & $\mathcal{B}\left(\times 10^{-6}\right)$ & $f_{L}$ & $C_{\text {long }}\left(A_{C P}\right)$ & $S_{\text {long }}$ \\
\hline$B^{0} \rightarrow \rho^{+} \rho^{-}$ & $B A B A R[30](82)$ & $30 \pm 4 \pm 5$ & $\cdots$ & $\cdots$ & $\cdots$ \\
$B^{0} \rightarrow \rho^{+} \rho^{-}$ & $B A B A R[26](210)$ & $\cdots$ & $0.978 \pm 0.014_{-0.029}^{+0.021}$ & $-0.03 \pm 0.18 \pm 0.09$ & $-0.33 \pm 0.24_{-0.14}^{+0.08}$ \\
$B^{0} \rightarrow \rho^{+} \rho^{-}$ & Belle [27] (253) & $22.8 \pm 3.8_{-2.6}^{+2.3}$ & $0.941_{-0.040}^{+0.034} \pm 0.030$ & $\cdots$ & $\cdots$ \\
$B^{0} \rightarrow \rho^{+} \rho^{-}$ & Belle [31] (535) & $\cdots$ & $\cdots$ & $-0.16 \pm 0.21 \pm 0.08^{\mathrm{a}}$ & $0.19 \pm 0.30 \pm 0.08$ \\
$B^{ \pm} \rightarrow \rho^{ \pm} \rho^{0}$ & $B A B A R[21](210)$ & $16.8 \pm 2.2 \pm 2.3$ & $0.905 \pm 0.042_{-0.027}^{+0.023}$ & $-0.12 \pm 0.13 \pm 0.10$ & $\cdots$ \\
$B^{ \pm} \rightarrow \rho^{ \pm} \rho^{0}$ & Belle [22] (78) & $31.7 \pm 7.1_{-6.7}^{+3.8}$ & $0.95 \pm 0.11 \pm 0.02$ & $0.00 \pm 0.22 \pm 0.03$ & $\cdots$ \\
$B^{0} \rightarrow \rho^{0} \rho^{0}$ & $B A B A R[23](349)$ & $1.07 \pm 0.33 \pm 0.19$ & $0.87 \pm 0.13 \pm 0.04$ & $\cdots$ & $\cdots$ \\
\hline \hline
\end{tabular}

${ }^{\mathrm{a}}$ Belle Collaboration uses the opposite sign convention for $C_{\mathrm{long}}$. 


$$
A_{C P}=\frac{\bar{N}-N}{\bar{N}+N}
$$

where $N(\bar{N})$ is the number of $B(\bar{B})$ decays to this final state. Table I summarizes the most recent measurements of the complete set of experimental inputs needed to extract $\alpha$ from the $B \rightarrow \rho \rho$ decays.

In this article, we present an update of the timedependent analysis of $B^{0} \rightarrow \rho^{+} \rho^{-}$and measurement of the CKM angle $\alpha$ reported in Ref. [26] and the branching fraction reported in Ref. [30]. Improvements to the analysis reported here include an increase in the data sample analyzed, a tighter constraint on the proper time difference between the two $B$ meson decays in selected events, an improved algorithm to determine the flavor of $B$ mesons, a modified multivariate analyzer for background suppression, and an improved description of the signal and background model.

\section{THE DATA SET AND BABAR DETECTOR}

The results presented in this paper are based on data collected with the BABAR detector at the PEP-II asymmetric $e^{+} e^{-}$storage ring [32] operating at the Stanford Linear Accelerator Center. At PEP-II, 9.0 GeV electrons and $3.1 \mathrm{GeV}$ positrons are collided at a center-of-mass energy of $10.58 \mathrm{GeV}$ which corresponds to the mass of the $Y(4 S)$ resonance. The asymmetric energies result in a boost from the laboratory to the center-of-mass (CM) frame of $\beta \gamma \approx$ 0.56 . The data set analyzed has an integrated luminosity of $349 \mathrm{fb}^{-1}$ corresponding to $(383.6 \pm 4.2) \times 10^{6} B \bar{B}$ pairs recorded at the $Y(4 S)$ resonance (on peak). An additional $27.2 \mathrm{fb}^{-1}$ of data were recorded about $40 \mathrm{MeV}$ below this energy (off peak) for the study of continuum background, where light quarks are produced in the process $e^{+} e^{-} \rightarrow q \bar{q}$ $(q=u, d, s, c)$.

The $B A B A R$ detector is described in detail elsewhere [33]. Surrounding the interaction point is a five doublesided layer silicon vertex tracker (SVT) which measures the impact parameters of charged particle tracks in both the plane transverse to and along the beam direction. A 40layer drift chamber (DCH) surrounds the SVT and provides measurements of the momenta for charged particles. Both the SVT and DCH are surrounded by a solenoid magnet that provides a $1.5 \mathrm{~T}$ magnetic field. Charged hadron identification is achieved through measurements of particle energy loss in the tracking system and the Cherenkov angle obtained from a detector of internally reflected Cherenkov light. A CsI(Tl) electromagnetic calorimeter (EMC) provides photon detection, electron identification, and $\pi^{0}$ reconstruction. Finally, the instrumented flux return of the magnet allows discrimination of muons from pions. For the most recent $134.0 \mathrm{fb}^{-1}$ of data, a portion of the resistive plate chambers constituting the muon system has been replaced by limited streamer tubes [34-36].
We use a right-handed coordinate system with the $z$ axis along the electron beam direction and the $y$ axis upward, with the origin at the nominal beam interaction point. Unless otherwise stated, kinematic quantities are calculated in the laboratory rest frame. The other reference frame which we commonly use is the CM frame of the colliding electrons and positrons.

We use Monte Carlo (MC) simulated events generated using the GEANT4 [37] based BABAR simulation.

\section{RECONSTRUCTION OF B CANDIDATES}

\section{A. Photon and $\pi^{0}$ reconstruction}

Photons are reconstructed from localized energy deposits in the EMC that are not associated with a charged track. We require photon candidates to have an energy greater than $50 \mathrm{MeV}$, and a lateral shower profile [38] to be consistent with the photon hypothesis. We reconstruct neutral pions from combinations of two distinct photon candidates where the invariant $\gamma \gamma$ mass $m_{\gamma \gamma}$ satisfies $0.10<m_{\gamma \gamma}<0.16 \mathrm{GeV} / c^{2}$.

\section{B. $\rho^{ \pm}$reconstruction}

We combine reconstructed $\pi^{0}$ mesons with charged tracks that are consistent with the $\pi^{ \pm}$hypothesis to form $\rho^{ \pm}$candidates. The invariant mass $m_{\pi^{ \pm} \pi^{0}}$ of the reconstructed $\rho^{ \pm}$is required to lie between 0.5 and $1.0 \mathrm{GeV} / c^{2}$, to select events in the vicinity of the $\rho$ resonance. We require that the helicity angle of each $\rho$ meson satisfies $-0.90<\cos \theta_{i}<0.98$. This selection criteria suppress continuum and $B$ backgrounds.

\section{C. $B^{0}$ reconstruction and event selection}

We combine two oppositely charged $\rho$ candidates to form the $B$ candidate $B_{\text {rec }}$. All particles in the rest of the event (ROE) are combined to form the other $B$ meson in the event $B_{\text {tag }}$. In addition to the two charged tracks in the $B_{\text {rec }}$, we require that there is at least one track in the $B_{\mathrm{tag}}$.

In order to suppress potential backgrounds from $e^{+} e^{-} \rightarrow e^{+} e^{-}, \mu^{+} \mu^{-}$events, we require the second-tozeroth Fox-Wolfram moment $R_{2}$ [39] of the event to be less than 0.98. Continuum events are the dominant background which is reduced by requiring the absolute value of the cosine of the angle between the $B_{\text {rec }}$ thrust axis $(T B)$ and that of the ROE $(T R)$ to satisfy $|\cos (T B, T R)|<0.8$. We retain $17.1 \%$ and $20.1 \%$ of longitudinal and transverse signal, respectively, by requiring the aforementioned selection criteria.

We calculate $\Delta t=\Delta z / \beta \gamma c$ from the measured separation $\Delta z$ between the $B_{\text {rec }}$ and $B_{\text {tag }}$ vertices [40]. We determine the $B_{\text {rec }}$ vertex from the two charged-pion tracks in its decay. The $B_{\text {tag }}$ decay vertex is obtained by fitting the other tracks in the event with constraints from the $B_{\text {rec }}$ momentum and the beam-spot location. The RMS resolution on $\Delta t$ 


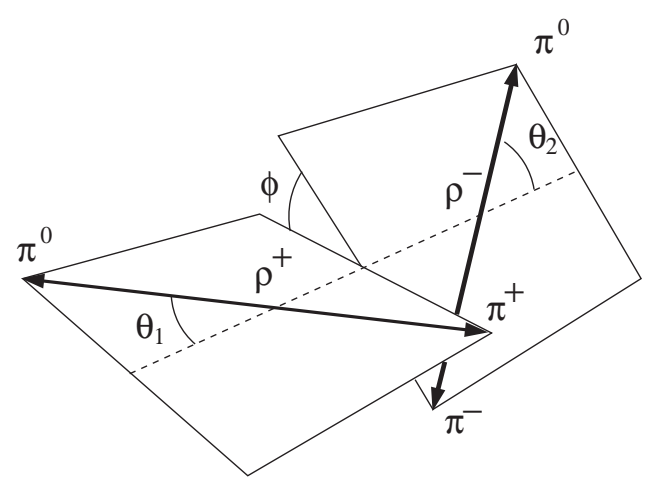

FIG. 3. A schematic view of the decay of a $B$ meson via two $\rho$ mesons to a four-pion final state. The $\rho$ meson final states are shown in their rest frames, and $\phi$ is the angle between the decay planes of the $\rho$ mesons.

is $1.1 \mathrm{ps}$. We only use events that satisfy $|\Delta t|<15 \mathrm{ps}$ and for which the error on $\Delta t\left(\sigma_{\Delta t}\right)$ is less than $2.5 \mathrm{ps}$.

Signal events are identified using two kinematic variables, the difference $\Delta E$ between the CM energy of the $B_{\text {rec }}$, $E_{B}^{*}$, and $\sqrt{s} / 2$,

$$
\Delta E=E_{B}^{*}-\sqrt{s} / 2
$$

and the beam-energy-substituted mass,

$$
m_{\mathrm{ES}}=\sqrt{\left(s / 2+\mathbf{p}_{i} \cdot \mathbf{p}_{B}\right)^{2} / E_{i}^{2}-\mathbf{p}_{B}^{2}}
$$

where $\sqrt{s}$ is the total CM energy. The $B_{\text {rec }}$ momentum $\mathbf{p}_{B}$ and four-momentum of the initial state $\left(E_{i}, \mathbf{p}_{i}\right)$ are defined
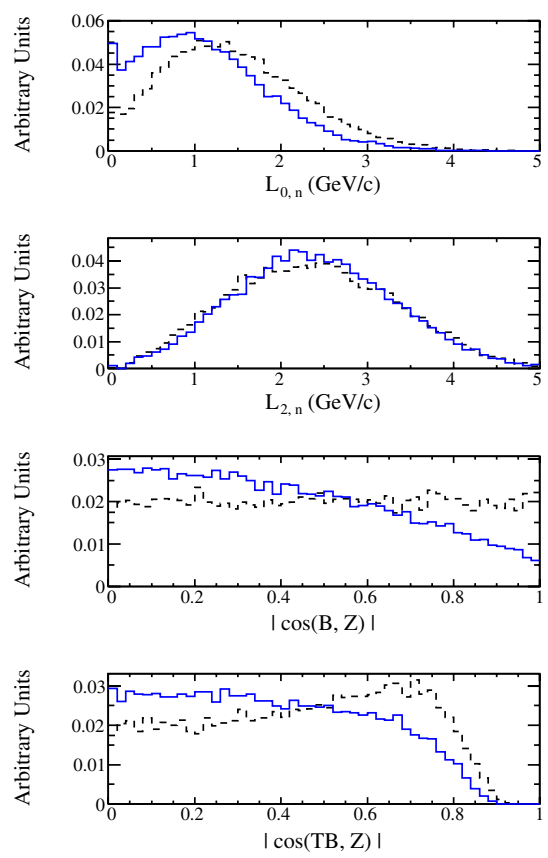

in the laboratory frame. We accept candidates that satisfy $5.25<m_{\mathrm{ES}}<5.29 \mathrm{GeV} / c^{2}$ and $-0.12<\Delta E<0.15 \mathrm{GeV}$. An asymmetric $\Delta E$ selection is used in order to reduce backgrounds from higher-multiplicity $B$ decays. The resolution on $m_{\mathrm{ES}}$ is dominated by the knowledge of the energy of the $e^{+}$and $e^{-}$beams, and the resolution on $\Delta E$ is dominated by the reconstruction performance of the EMC.

After the selection criteria mentioned above have been applied, the average number of candidates per event is approximately 1.5 . In events with more than one candidate, we select the candidate that minimizes the sum of $\left(m_{\gamma \gamma}-\right.$ $\left.m_{\pi^{0}}\right)^{2}$ where $m_{\pi^{0}}$ is the true $\pi^{0}$ mass [28]. In $0.3 \%$ of events, the same $\pi^{0}$ mesons are used by multiple $B$ candidates. In such an event we randomly select the candidate to keep.

\section{CONTINUUM BACKGROUND SUPPRESSION}

In addition to the cuts on $\cos \theta_{i}, R_{2}$, and $|\cos (T B, T R)|$ that directly remove background events, we use an artificial neural network in order to discriminate between continuum background and signal events. For this purpose we combine the following eight variables into a single output, $\mathcal{N}$.

(i) The coefficients, $L_{0}, L_{2}$, split into sums over the ROE for neutral and charged particles: $L_{0, n}, L_{2, n}$ and $L_{0, c}, L_{2, c}$. The coefficients are defined as $L_{k}=$ $\sum p_{j}\left|\cos \left(\psi_{j}\right)\right|^{k}$, where $k=0,2, p_{j}$ is the particle momentum, and $\psi_{j}$ is the angle of the particle direction relative to the thrust axis of the $B$ candidate. Both $p_{j}$ and $\psi_{j}$ are defined in the CM frame.
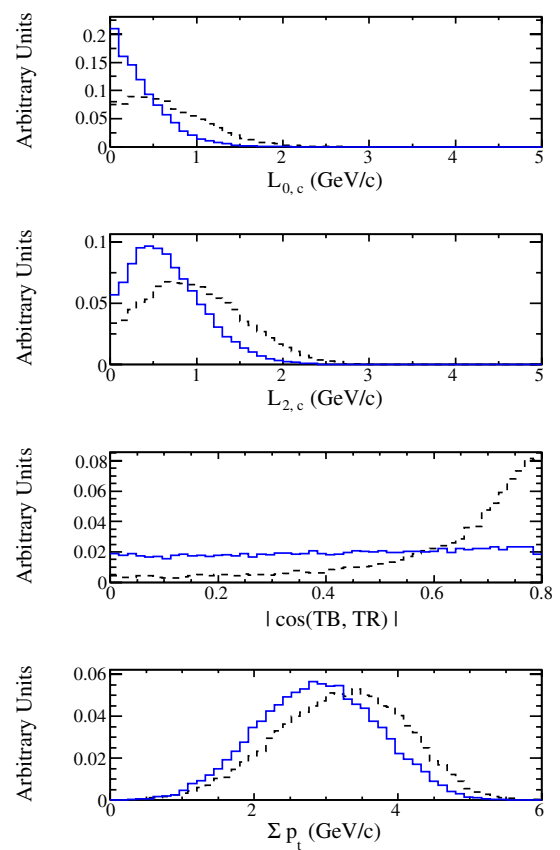

FIG. 4 (color online). The input variables used in training the neural network. The solid line represents the signal training sample (MC simulated events) and the dashed line represents the continuum background (off-peak data). The distributions shown are (in order from left to right; top to bottom) $L_{0, n}, L_{0, c}, L_{2, n}, L_{2, c}, \cos (B, Z), \cos (B, T R), \cos (T B, Z)$, and the sum of the $p_{t}$ in the ROE. 

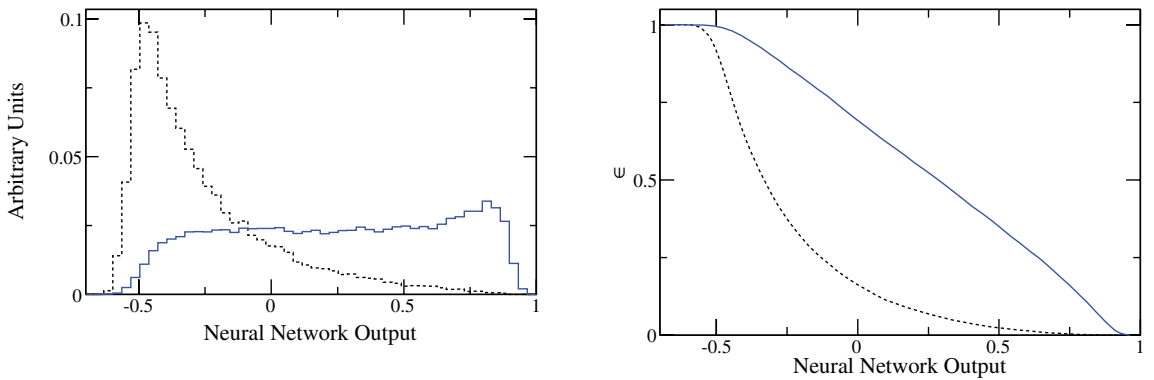

FIG. 5 (color online). The left plot shows the distribution of $\mathcal{N}$ for off-peak data (dashed line) and longitudinally polarized signal MC simulated events (solid line). The right plot shows the signal efficiency (solid line) and continuum background efficiency (dashed line) distribution as a function of cut on the minimum value of $\mathcal{N}$.

(ii) $|\cos (B, Z)|$, the absolute value of the cosine of the angle between the direction of the $B$ and $z$ axis in the $\mathrm{CM}$ frame. This variable follows a sine squared distribution for $B \bar{B}$ events, whereas it is almost uniform for $q \bar{q}$.

(iii) $|\cos (T B, T R)|$. This variable, previously defined in Sec. III C, is strongly peaked at unity for $q \bar{q}$ events. $B \bar{B}$ events are more isotropic as the $B$ mesons are produced close to the kinematic threshold.

(iv) $|\cos (T B, Z)|$, the absolute value of the cosine of the angle between the $B$ thrust and the $z$ axis.

(v) The scalar sum of the transverse momenta $p_{t}$ in the ROE. This sum includes neutral and charged particles.

The distributions of these input variables are shown in Fig. 4. Figure 5 shows $\mathcal{N}$ for signal MC simulated events and continuum background samples (off-peak data) and the efficiency for signal and $q \bar{q}$ background as a function of cut on the minimum value of $\mathcal{N}$. We require $\mathcal{N}$ to be greater than -0.4 . Note that later we use this variable in the maximum likelihood fit described in Sec. VII.

The samples used to train the neural net were correctly reconstructed MC simulated events and off-peak data. To avoid overtraining, we used an independent sample of these data (i.e. distinct from the sample used for the training) to check the performance of the network. The training is stopped when the separation between the signal and continuum background is stable.

\section{MISRECONSTRUCTED SIGNAL AND SELECTION EFFICIENCY}

Misreconstructed signal candidates, referred to as selfcross-feed (SCF) signal, may pass the selection requirements even if one or more of the pions assigned to the $\rho^{+} \rho^{-}$state belongs to the other $B$ in the event. These SCF candidates constitute $50.7 \%$ (27.9\%) of the accepted longitudinally (transversely) polarized signal. The majority of SCF events have both charged pions from the $\rho^{+} \rho^{-}$final state and unbiased $C P$ information. These correct (right) track SCF events are denoted by RT SCF. There is a SCF component (13.8\% of the signal) where at least one track in
$B_{\text {rec }}$ is from the ROE. These wrong track (WT) events are used to determine the signal yield $N_{\text {signal }}$ and $f_{L}$ but have biased $C P$ information, and are treated separately for the $C P$ result. A systematic error is assigned to the $C P$ results from this type of signal event. The total selection efficiency for longitudinally (transversely) polarized signal is $7.7 \%$ $(10.5 \%)$.

\section{VI. $B$ MESON FLAVOR TAGGING}

A key ingredient in the measurement of time-dependent $C P$ asymmetries is to determine whether, at the time of decay of the $B_{\text {tag }}$, corresponding to $\Delta t=0$, the $B_{\text {rec }}$ was a $B^{0}$ or a $\bar{B}^{0}$. This "flavor tagging" is achieved with the analysis of the decay products of the recoiling $B$ meson $B_{\text {tag }}$. The overwhelming majority of $B$ mesons decay to a final state that is flavor specific, i.e. only accessible from either a $B^{0}$ or a $\bar{B}^{0}$, but not from both. The purpose of the flavor tagging algorithm is to determine the flavor of $B_{\text {tag }}$ with the highest possible efficiency $\epsilon_{\text {tag }}$ and lowest possible probability $w$ of assigning a wrong flavor to $B_{\text {tag }}$. It is not necessary to fully reconstruct $B_{\text {tag }}$ in order to determine its flavor. In the presence of a finite mistag probability $w$, the $C P$ asymmetry is reduced by a dilution factor $1-2 w$, so Eq. (7) becomes

$$
\begin{aligned}
f_{ \pm}(\Delta t)= & \frac{e^{-|\Delta t| / \tau_{B^{0}}}}{4 \tau_{B^{0}}}\left\{1 \pm(1-2 w) \eta_{f}\left[S \sin \left(\Delta m_{d} \Delta t\right)\right.\right. \\
& \left.\left.-C \cos \left(\Delta m_{d} \Delta t\right)\right]\right\} .
\end{aligned}
$$

The figure of merit for the performance of the tagging algorithm is the effective tagging efficiency

$$
Q=\epsilon_{\mathrm{tag}}(1-2 w)^{2},
$$

which is related to the statistical uncertainty $\sigma$ in the coefficients $S$ and $C$ through

$$
\sigma \propto \frac{1}{\sqrt{Q}} .
$$

We use a neural network based technique $[11,40]$ that isolates primary leptons, kaons, and pions from $B$ decays to final states containing $D^{*}$ mesons, and high momentum 
TABLE II. Tagging efficiency $\epsilon$, average mistag fraction $w$, mistag fraction difference between $B^{0}$ and $\bar{B}^{0}$ tagged events $\Delta w$, and $Q$ for $B^{0} \rightarrow \rho^{+} \rho^{-}$events.

\begin{tabular}{l|rrrr}
\hline \hline Category & \multicolumn{1}{|c}{$\epsilon_{\mathrm{tag}}(\%)$} & \multicolumn{1}{c}{$\omega(\%)$} & $\Delta \omega(\%)$ & \multicolumn{1}{c}{$Q(\%)$} \\
\hline Lepton & $8.2 \pm 0.1$ & $3.2 \pm 0.5$ & $-0.2 \pm 0.8$ & $7.2 \pm 0.2$ \\
Kaon I & $11.3 \pm 0.1$ & $3.7 \pm 0.7$ & $1.1 \pm 1.2$ & $9.7 \pm 0.3$ \\
Kaon II & $17.3 \pm 0.2$ & $14.2 \pm 0.7$ & $-0.9 \pm 1.1$ & $8.8 \pm 0.3$ \\
Kaon-Pion & $13.4 \pm 0.1$ & $20.8 \pm 0.8$ & $0.5 \pm 1.3$ & $4.6 \pm 0.3$ \\
Pion & $13.8 \pm 0.2$ & $30.6 \pm 0.8$ & $4.1 \pm 1.3$ & $2.1 \pm 0.2$ \\
Other & $9.4 \pm 0.1$ & $40.1 \pm 1.0$ & $2.3 \pm 1.5$ & $0.4 \pm 0.1$ \\
Untagged & $26.8 \pm 0.2$ & $50.0 \pm 0.0$ & $\cdots$ & $0.0 \pm 0.0$ \\
Total & & & & $32.7 \pm 0.7$ \\
\hline
\end{tabular}

charged particles from $B$ decays, to determine the flavor of the $B_{\text {tag }}$. The output of this algorithm is divided into seven mutually exclusive categories. These are (in order of decreasing signal purity) Lepton, Kaon I, Kaon II, KaonPion, Pion, Other, and Untagged. The performance of this algorithm is determined using fully reconstructed neutral $B$ decays to $D^{(*)-}\left(\pi^{+}, \rho^{+}, a_{1}^{+}\right)$and is summarized in Table II. The categories assigned correspond to events with leptons, kaons, and pions in the decay products of $B_{\text {tag }}$. The untagged events contain no flavor information and therefore carry no weight in the time-dependent analysis. In addition, these events are not considered useful for the branching fraction measurement since they are dominated by continuum background. The total $Q$ of this algorithm is $32.7 \pm 0.7 \%$.

\section{LIKELIHOOD FIT METHOD}

On applying the selection criteria described above, we obtain a sample of 37424 events that enter the fit. These events are dominated by backgrounds from $q \bar{q}(81.4 \%)$ and $B \bar{B}(16.6 \%)$ events. The remaining $2 \%$ of events are considered as signal. We distinguish between the following components in the fit:

(i) correctly reconstructed signal,

(ii) SCF signal, split into RT and WT parts,

(iii) charm $B^{ \pm}$backgrounds $(b \rightarrow c)$,

(iv) charm $B^{0}$ backgrounds $(b \rightarrow c)$,

(v) charmless $B^{0}$ backgrounds,

(vi) charmless $B^{ \pm}$backgrounds,

(vii) continuum background.

The dominant $B$ backgrounds come from components (iii) and (iv). The signal, continuum, and $B$ background models are described in Secs. VII A, VII B, and VII C, respectively.

We use an unbinned, extended maximum likelihood (ML) fit to extract $N_{\text {signal }}, f_{L}, S_{\text {long }}$, and $C_{\text {long }}$. The likelihood function incorporates the following previously defined eight discriminating variables to distinguish signal from the backgrounds: $m_{\mathrm{ES}}, \Delta E, \Delta t, \mathcal{N}$, and the $m_{k}$ and $\cos \theta_{k}$ values of the two $\rho$ mesons, where $k=1,2$. For each of the aforementioned components $j$, we construct a probability density function (PDF) that is the product of one- dimensional PDFs for each of the variables. The PDFs do not account for all possible correlations among the discriminating variables, and we account for possible biases as a result of neglecting these correlations as discussed in Sec. VIII. For each event $i$, the PDFs can be written as

$$
\begin{aligned}
\mathcal{P}_{j}^{i}= & \mathcal{P}_{j}\left(m_{\mathrm{ES}}^{i}\right) \mathcal{P}_{j}\left(\Delta E^{i}\right) \mathcal{P}_{j}\left(\Delta t^{i}\right) \mathcal{P}_{j}\left(\mathcal{N}^{i}\right) \mathcal{P}_{j}\left(m_{1}^{i}\right) \mathcal{P}_{j}\left(m_{2}^{i}\right) \\
& \times \mathcal{P}_{j}\left(\cos \theta_{1}^{i}\right) \mathcal{P}_{j}\left(\cos \theta_{2}^{i}\right)
\end{aligned}
$$

We determine the PDFs for signal and each of the $B$ background components from MC distributions for each discriminating variable. The PDFs for the continuum background are determined from on-peak and off-peak data. The likelihood function is

$$
\mathcal{L}=\frac{\exp \left(-\sum_{j} n_{j}\right)}{N !} \prod_{i}^{N} \sum_{j} n_{j} \mathcal{P}_{j}^{i},
$$

where $n_{j}$ are the event yields of hypotheses $j$ (determined from the ML fit) and $N$ is the observed number of events in the sample. The normalized exponent takes into account Poisson fluctuations in the total number of events. We simultaneously fit events in the mutually exclusive flavor tag categories listed in Table II, excluding events in the untagged category. There are six continuum background yields, one for each flavor tag category, and we use a single yield for the signal and each of the $B$ backgrounds, while accounting for the relative fractions of events expected in each of the flavor tag categories. The fit results are obtained by minimizing the value of $-\ln \mathcal{L}$.

\section{A. Signal model}

The signal has contributions from true and SCF longitudinally (long) and transversely (tran) polarized events. In addition to this, the longitudinally polarized SCF signal PDF is further subdivided into the two categories of misreconstructed signal: RT and WT SCF signal, and all signal PDFs are subdivided according to the $B_{\text {tag }}$ flavor tag category. The total signal PDF is given by

$$
\begin{aligned}
\mathcal{P}_{\text {signal }}= & f_{L}\left(\left[1-f_{\mathrm{RT} \mathrm{SCF}}^{\text {long }}-f_{\mathrm{WT} \mathrm{SCF}}^{\text {long }}\right] \mathcal{P}_{\text {true }}^{\text {long }}\right. \\
& \left.+f_{\mathrm{RT} \mathrm{SCF}}^{\text {long }} \mathcal{P}_{\mathrm{RT} \text { SCF }}^{\text {long }}+f_{\mathrm{WT} \mathrm{SCF}}^{\text {long }} \mathcal{P}_{\mathrm{WT} \text { SCF }}^{\text {long }}\right) \\
& +\left(1-f_{L}\right)\left(\left[1-f_{\mathrm{SCF}}^{\text {tran }}\right] \mathcal{P}_{\text {true }}^{\text {tran }}+f_{\mathrm{SCF}}^{\text {tran }} \mathcal{P}_{\mathrm{SCF}}^{\text {tran }}\right),
\end{aligned}
$$

where $f_{\mathrm{RT}(\mathrm{WT}) \mathrm{SCF}}^{\text {long }}$ is the fraction of longitudinally polarized RT (WT) SCF signal and $f_{\text {SCF }}^{\text {tran }}$ is the fraction of transversely polarized SCF signal. The PDFs $\mathcal{P}_{\mathrm{RT}(\mathrm{WT}) \mathrm{SCF}}^{\text {long }}$ and $\mathcal{P}_{\text {SCF }}^{\text {tran }}$ are defined accordingly. In order to extract the observable $f_{L}$ from the fit, we account for the different reconstruction efficiencies for longitudinally and transversely polarized signal using

$$
f_{L}=\frac{f_{L}^{\mathrm{obs}}}{R+f_{L}^{\mathrm{obs}}(1-R)},
$$


where $f_{L}^{\text {obs }}$ is the fraction of signal from longitudinally polarized events in the data, and $R$ is the ratio of longitudinal to transverse polarized signal efficiencies.

All of the SCF PDFs are further subdivided into parts that contain correctly reconstructed and misreconstructed $\rho$ mesons. Four subdivisions are used, corresponding to both mesons being correctly reconstructed (TT), one being correctly reconstructed and the other being misreconstructed ( $T F$ or $F T$ ), and both mesons being misreconstructed $(F F)$. Here the first (second) $\rho$ meson is positively (negatively) charged. The SCF PDFs have the form

$$
\begin{aligned}
\mathcal{P}= & \mathcal{P}(\mathcal{N}) \mathcal{P}(\Delta t) \\
& \times\left[f_{T T} \mathcal{P}_{T T}\left(m_{\mathrm{ES}}, \Delta E, \cos \theta_{1}, m_{1}, \cos \theta_{2}, m_{2}\right)\right. \\
& +f_{T F} \mathcal{P}_{T F}\left(m_{\mathrm{ES}}, \Delta E, \cos \theta_{1}, m_{1}, \cos \theta_{2}, m_{2}\right) \\
& +f_{F T} \mathcal{P}_{F T}\left(m_{\mathrm{ES}}, \Delta E, \cos \theta_{1}, m_{1}, \cos \theta_{2}, m_{2}\right) \\
& \left.+f_{F F} \mathcal{P}_{F F}\left(m_{\mathrm{ES}}, \Delta E, \cos \theta_{1}, m_{1}, \cos \theta_{2}, m_{2}\right)\right],
\end{aligned}
$$

where $T$ and $F$ are correctly and misreconstructed $\rho$ mesons, respectively, $f_{T T}, f_{T F}, f_{F T}, f_{F F}$ are the fractions of each type of misreconstructed event, and the PDFs for $m_{\mathrm{ES}}$, $\Delta E$, and the helicity and mass for each reconstructed $\rho$ meson are the products of one-dimensional PDFs. The signal PDFs for $\mathcal{N}$ and $\Delta t$ have different shapes according to the $B_{\text {tag }}$ flavor tag category assigned to an event.

The longitudinally polarized true and RT SCF signal $m_{\mathrm{ES}}$ distributions are described by a Gaussian with a power law tail [41-43] which takes the following form:

$$
\begin{aligned}
f(m) & \propto \exp \left(-\frac{\left(m-m_{0}\right)^{2}}{2 \sigma^{2}}\right), & & m>m_{0}-\xi \sigma, \\
& \propto \frac{n^{n} \exp \left(-\xi^{2} / 2\right)}{\left(\frac{\xi\left(m_{0}-m\right)}{\sigma}+n-\xi^{2}\right)^{n}}, & & \leq m_{0}-\xi \sigma,
\end{aligned}
$$

where $m_{0}$ is the mean, $\sigma$ is the width of the Gaussian part, and the parameters $\xi$ and $n$ describe the exponential tail. The remaining PDFs for the signal $m_{\mathrm{ES}}$ distributions are the sum of a Gaussian and a Gaussian with an exponential tail. The signal $\Delta E$ distributions for the longitudinally polarized SCF events are described by first and second order polynomials, with the exception of $T T$ events, which are modeled with the sum of a second order polynomial and a Gaussian. The other $\Delta E$ PDFs are described by the sum of a Gaussian and a Gaussian with an exponential tail. The $\mathcal{N}$ distributions are modeled using smoothed histograms of MC simulated events. The signal $\Delta t$ distribution is described by Eq. (16) convolved with a triple Gaussian resolution function given by

$$
\begin{aligned}
\mathcal{R}_{\text {sig }}\left(\Delta t, \sigma_{\Delta t}\right)= & f_{\text {core }} G\left(\Delta t, \mu_{\text {core }} \sigma_{\Delta t}, \sigma_{\text {core }} \sigma_{\Delta t}\right) \\
& +f_{\text {tail }} G\left(\Delta t, \mu_{\text {tail }} \sigma_{\Delta t}, \sigma_{\text {tail }} \sigma_{\Delta t}\right) \\
& +f_{\text {outlier }} G\left(\Delta t, \mu_{\text {outlier }}, \sigma_{\text {outlier }}\right),
\end{aligned}
$$

where $G$ is a Gaussian with mean $\mu_{l}$ and width $\sigma_{l}$ for $l=$ core, tail, and outlier. The parameters $\sigma_{\text {tail }}, \sigma_{\text {outlier }}$, and $\mu_{\text {outlier }}$ are set to $3.0 \mathrm{ps}, 8.0 \mathrm{ps}$, and $0.0 \mathrm{ps}$, respectively. The remaining parameters of the resolution function are determined from signal MC simulated events scaled by the differences between data and MC simulated events for large samples of exclusively reconstructed $B$ decays to $D^{(*)-}\left(\pi^{+}, \rho^{+}, a_{1}^{+}\right)$final states described in Sec. VI. The values of the mean and width of the core Gaussian contribution to the resolution function depend on the flavor tagging category of an event. There are three signal $\Delta t$ PDFs, one for the true and RT SCF longitudinally polarized signal, one for the WT SCF longitudinally polarized signal, and one for the transversely polarized signal. Each of these PDFs has distinct values of $S$ and $C$ as described in Secs. I and V. The WT SCF longitudinally polarized signal has a different resolution function with respect to the rest of the signal. The $\cos \theta_{i}$ distribution for true signal events is described by the expected cosine squared or sine squared distribution multiplied by a polynomial acceptance function. The $\cos \theta_{i}$ PDFs for $T \rho$ mesons in longitudinally (transversely) polarized SCF signal are described by sixth order polynomials (smoothed histograms of MC simulated events). The $\cos \theta_{i}$ PDFs for misreconstructed $\rho$ mesons are described by smoothed histograms of MC simulated events. The longitudinally polarized signal $\rho$ mass PDFs are described using relativistic Breit-Wigner function and third order polynomials for $T$ and $F \rho$ contributions, respectively. The transversely polarized signal SCF $\rho$ mass PDF distributions are described using smoothed histograms of MC simulated events.

In addition to $N_{\text {signal }}, f_{L}, S_{\text {long }}$, and $C_{\text {long }}$, we determine the mean of the core Gaussian part of the $m_{\mathrm{ES}}$ and $\Delta E$ distributions from the fit. We obtain parameters consistent with expectations from MC simulated events.

\section{B. Continuum background model}

The continuum background PDFs are also subdivided according to the misreconstruction of $\rho$ mesons in the final state and have the form

$$
\begin{aligned}
\mathcal{P}= & \mathcal{P}\left(m_{\mathrm{ES}}\right) \mathcal{P}(\Delta E) \mathcal{P}(\mathcal{N}) \mathcal{P}(\Delta t) \\
& \times\left[f_{T T} \mathcal{P}_{T T}\left(\cos \theta_{1}, m_{1}, \cos \theta_{2}, m_{2}\right)\right. \\
& +f_{T F} \mathcal{P}_{T F}\left(\cos \theta_{1}, m_{1}, \cos \theta_{2}, m_{2}\right) \\
& +f_{F T} \mathcal{P}_{F T}\left(\cos \theta_{1}, m_{1}, \cos \theta_{2}, m_{2}\right) \\
& \left.+f_{F F} \mathcal{P}_{F F}\left(\cos \theta_{1}, m_{1}, \cos \theta_{2}, m_{2}\right)\right]
\end{aligned}
$$

where helicity and mass PDFs for each reconstructed $\rho$ meson are the products of one-dimensional PDFs.

The continuum distribution for $m_{\mathrm{ES}}$ is described by a phase-space-motivated distribution [44] with the following form:

$$
f(x) \propto x \sqrt{1-x^{2}} \cdot \exp \left[\xi\left(1-x^{2}\right)\right] \cdot \theta\left(m_{\mathrm{ES}}\right),
$$


where $\theta\left(m_{\mathrm{ES}}\right)=1$ for $m_{\mathrm{ES}} \leq \sqrt{s} / 2$ and $\theta\left(m_{\mathrm{ES}}\right)=0$ for $m_{\mathrm{ES}}>\sqrt{s} / 2$ and $x=2 m_{\mathrm{ES}} / \sqrt{s}$. The $\Delta E$ and $\mathcal{N}$ shapes are modeled with third and fourth order polynomials, respectively. The parameters of the $m_{\mathrm{ES}}, \Delta E$, and $\mathcal{N}$ shapes are allowed to vary in the fit to the on-peak data. The continuum $\rho$ mass distribution is described using a relativistic Breit-Wigner function and a third order polynomial PDF for $T$ and $F \rho$ contributions, respectively. The $\cos \theta_{i}$ distribution is described by a third order polynomial. The continuum $\Delta t$ distribution has a prompt lifetime component convolved with a triple Gaussian resolution function. The parameters of the $\rho$ mass and helicity distributions are obtained from a fit to the off-peak data, and the remaining parameters are determined in the fit.

\section{C. $B$ background model \\ 1. Charm B backgrounds}

Combinatorial events from $b \rightarrow c$ transitions are the dominant $B$ backgrounds. These components have shapes similar to continuum and do not peak in the signal region for the discriminating variables. The functional form used for the PDFs of these background components is given by Eq. (26). We parametrize the $m_{\mathrm{ES}}$ and $\Delta E$ distributions of these backgrounds using the phase-space-motivated distribution of Eq. (27), and a third order polynomial, respectively. The $\rho$ mass distribution is described using a relativistic Breit-Wigner function and a third order polynomial PDF for $T$ and $F \rho$ contributions, respectively. The remaining PDFs are described using smoothed histograms of MC simulated events. Each of the PDF parameters for these backgrounds are determined from samples of $\mathrm{MC}$ simulated events, and the yields of these components are determined in the fit. When studying systematic uncertainties, we modify the PDF used for $\Delta t$ so that it has a form similar to the signal which uses the resolution function of Eq. (25). An effective lifetime that is smaller than $\tau_{B^{0}}$ is used to account for misreconstruction of these events and the finite charm meson lifetime. The value of this parameter is obtained by fitting MC simulated events for this category of events.

\section{Charmless B backgrounds}

Some of the charmless $B$ backgrounds have PDFs similar to the signal for one or more of the discriminating variables, so it is important to correctly account for such events in the fit. We consider the 20 exclusive and two inclusive components of this type of $B$ background listed in Table III. If a charmless $B$ background decay contributes an event yield more than $1 \%$ of the expected signal yield, we model that mode exclusively. The remaining 140 charmless background components considered were combined with the appropriate weightings to form the neutral and charged inclusive charmless $B$ background components. Where possible, we use branching fractions from
TABLE III. The components of charmless $B$ backgrounds considered, along with the branching fraction $(\mathcal{B})$ and number of events $\left(N_{\mathrm{bg}}\right)$ expected in the selected data sample, where $†$ indicates a longitudinally polarized final state, and NR denotes a nonresonant final state. Where appropriate, branching fractions of these decays are quoted, including the branching fraction of subdecay modes and measured or expected values of $f_{L}$.

\begin{tabular}{l|cc}
\hline \hline Decay mode & $\mathcal{B} \times 10^{-6}$ & $N_{\mathrm{bg}}$ \\
\hline$B^{+} \rightarrow K^{+} \pi^{+} \pi^{-}[45,46]$ & $55 \pm 3$ & $11 \pm 1$ \\
$B^{+} \rightarrow \pi^{+} \pi^{0} \pi^{0}$ & $10 \pm 10$ & $15 \pm 15$ \\
$B^{+} \rightarrow \pi^{0} \rho^{+}[47-49]$ & $11 \pm 2$ & $73 \pm 10$ \\
$B^{+} \rightarrow \rho^{+} \rho^{0}[21,22] \dagger$ & $17 \pm 3$ & $71 \pm 12$ \\
$B^{+} \rightarrow \rho^{+} \pi^{+} \pi^{-}$ & $10 \pm 10$ & $9 \pm 8$ \\
$B^{+} \rightarrow K_{2}^{*}(1770) \rho$ & $10 \pm 10$ & $9 \pm 9$ \\
$B^{+} \rightarrow K_{0}^{*} \pi[45,46,49]$ & $11 \pm 1$ & $9 \pm 1$ \\
$B^{+} \rightarrow a_{1}^{+} \pi^{0}$ & $20 \pm 20$ & $60 \pm 60$ \\
$B^{+} \rightarrow \rho^{+} \pi^{0} \pi^{0}$ & $10 \pm 10$ & $12 \pm 12$ \\
$B^{+} \rightarrow a_{1}^{0}\left(\rho^{+} \pi^{-}\right) \rho^{+} \dagger$ & $8 \pm 8$ & $13 \pm 13$ \\
$B^{+} \rightarrow a_{1}^{0} \pi^{+}$ & $20 \pm 20$ & $49 \pm 49$ \\
$B^{+} \rightarrow \operatorname{charmless}$ & $30 \pm 3$ & $59 \pm 15$ \\
$B^{0} \rightarrow \pi^{+} \pi^{-} \pi^{0}(\mathrm{NR})$ & $24 \pm 3$ & $23 \pm 23$ \\
$B^{0} \rightarrow \rho^{ \pm} \pi^{\mp}[49,50]$ & $23 \pm 23$ & $42 \pm 4$ \\
$B^{0} \rightarrow \pi^{0} \pi^{0} K_{S}^{0}$ & $20 \pm 2$ & $15 \pm 15$ \\
$B^{0} \rightarrow a_{1}^{ \pm}\left(\rho^{0} \pi^{ \pm}\right) \pi^{\mp}[51]$ & $10 \pm 10$ & $7 \pm 1$ \\
$B^{0} \rightarrow \rho^{+} \pi^{ \pm} \pi^{0}$ & $10 \pm 10$ & $45 \pm 45$ \\
$B^{0} \rightarrow K_{2}^{*}(1770) \rho$ & $16 \pm 16$ & $8 \pm 8$ \\
$B^{0} \rightarrow a_{1}^{ \pm}\left(\rho^{ \pm} \pi^{0}\right) \rho^{\mp}[52] \dagger$ & $16 \pm 15$ & $43 \pm 43$ \\
$B^{0} \rightarrow a_{1}^{ \pm}\left(\rho^{0} \pi^{ \pm}\right) \rho^{\mp}[52] \dagger$ & $40 \pm 4$ & $9 \pm 8$ \\
$B^{0} \rightarrow a_{1}^{ \pm}\left(\rho^{ \pm} \pi^{0}\right) \pi^{\mp}[51]$ & $\cdots$ & $102 \pm 9$ \\
$B^{0} \rightarrow \operatorname{charmless}$ & & $88 \pm 22$ \\
\hline \hline
\end{tabular}

existing measurements. Where measurements are not available, we have either tried to use $S U(2)$ and $S U(3)$ flavor symmetries to relate an unmeasured decay to a measured one or, where this is not possible, we have assigned a branching fraction of $10 \times 10^{-6}$ to a decay mode. An uncertainty of $100 \%$ is assumed on all extrapolated branching fractions. We assume that the $a_{1}$ meson decays into a three pion final state via $\rho \pi$. The decay $B^{0} \rightarrow$ $a_{1}^{0} \pi^{0}$ is penguin dominated and is expected to have a small branching fraction, as is the case for the penguin dominated decays $B^{0} \rightarrow \rho^{0} \pi^{0}$ and $B^{0} \rightarrow \rho^{0} \rho^{0}$. As a result, we have neglected possible contributions from this potential source of background. Although we do not see evidence for $B^{0} \rightarrow \rho^{\mp} \pi^{ \pm} \pi^{0}$, we do consider this channel as a potential source of background. Contribution from $B^{0} \rightarrow 4 \pi$ is expected to be even smaller and is neglected. When considering the systematic uncertainty arising from interference between signal and other $\pi^{+} \pi^{-} \pi^{0} \pi^{0}$ final states, we assume that the nonresonant $\pi^{+} \pi^{-} \pi^{0} \pi^{0}$ final state has the same branching fraction as that of $B^{0} \rightarrow \rho^{\mp} \pi^{ \pm} \pi^{0}$, where $\mathcal{B}\left(B^{0} \rightarrow \rho^{\mp} \pi^{ \pm} \pi^{0}\right)$ is calculated from the yield determined in the $B^{0} \rightarrow \rho^{+} \rho^{-}$nominal fit. This is discussed further in the Appendix.

The functional form used for the PDFs of these charmless $B$ background components is given by Eq. (26). The 
$\Delta E$ distributions are described by third order polynomials, except for nonresonant $B^{0} \rightarrow \rho^{\mp} \pi^{ \pm} \pi^{0}$ which uses smoothed histograms of $\mathrm{MC}$ simulated events. The $m_{\rho}$ distributions for true $\rho$ mesons are parametrized using a relativistic Breit-Wigner function, and the fake $m_{\rho}$ (combinatorial $\pi^{ \pm} \pi^{0}$ ) distribution is described using third order polynomials. The remaining background shapes are described using smoothed histograms of MC simulated events. The yield for $B^{0} \rightarrow \rho^{\mp} \pi^{ \pm} \pi^{0}$ decays is allowed to vary in the fit. All other charmless background yields are fixed to expectations. This constraint is relaxed when studying possible sources of systematic uncertainties. When studying systematic uncertainties from possible $C P$ violation in the $B$ background, we modify the PDF used for $\Delta t$ so that it has a form similar to the signal which uses the signal resolution function in Eq. (25).

\section{LIKELIHOOD FIT VALIDATION}

Before applying the fitting procedure to the data, we subject it to various tests. The aim of these tests is to verify that one can extract the signal observables $N_{\text {signal }}, f_{L}, S_{\text {long }}$, and $C_{\text {long }}$ in a controlled way. Consistency of the likelihood fit is checked with ensembles of experiments simulated from the PDFs. The event yields are generated according to a Poisson distribution with mean $n_{j}$ for each category in these ensembles. In each of these tests we verify that the values generated for the signal observables are reproduced with the expected resolution. The distribution of $-\ln \mathcal{L}$ for the ensemble of experiments in comparison to that obtained when fitting the data provides an additional, but limited, cross-check of the consistency.

The PDFs used in the likelihood do not account for all possible correlations among the discriminating variables. We account for possible biases as a result of neglecting these correlations by fitting ensembles of experiments obtained from samples of signal and the charmless $B$ background MC simulated events combined with charm backgrounds and $q \bar{q}$ background events simulated from the PDFs. The MC simulated events used in these ensembles have these correlations modeled. We find a positive bias of $38.5 \pm 5.3$ events on $N_{\text {signal }}$, and a negative bias of $0.016 \pm$ 0.002 on $f_{L}$, and we do not observe a significant bias on $S_{\text {long }}$ and $C_{\text {long }}$.

As continuum events are the dominant background, we apply the fitting procedure to the off-peak data (after correcting for the difference in $\sqrt{s}$ ) to confirm that we do not find a fake signal in this control sample of events. We fit 790 off-peak events and extract signal and continuum yields of $8 \pm 7$ and $782 \pm 28$ events, respectively.

A blind analysis technique has been used for the extraction of $S_{\text {long }}$ and $C_{\text {long }}$, where the actual values of these observables have been hidden by an offset. With the values of $S_{\text {long }}$ and $C_{\text {long }}$ hidden, we perform the following fit cross-checks. We first verify that the uncertainties on the signal observables, and the value of $-\ln \mathcal{L}$ obtained from the fit to data, are compatible with the ensembles of simulated experiments described above. We then validate the stability of our results by introducing a variety of modifications to the fitting procedure. In addition to the nominal set of variables determined from data, we allow the physics parameters $\tau_{B^{0}}$ and $\Delta m_{d}$ to vary in turn. The signal observables do not change significantly when doing this, and the results obtained for $\tau_{B^{0}}$ and $\Delta m_{d}$ are $1.72 \pm 0.16 \mathrm{ps}$ and $0.36 \pm 0.22 \hbar / \mathrm{ps}$, respectively, consistent with the reported world average [28].

The Lepton and Kaon I tagged events have the highest signal purity, and lowest $w$ probability, and dominate our $C P$ results. We check that the results obtained from fitting only these categories of events are compatible with our nominal blind results. When doing this, we observe shifts of $-0.08(-0.05)$ on $S_{\text {long }}\left(C_{\text {long }}\right)$ relative to the nominal result.

Given that there are a number of $B$ backgrounds that contribute to the data that have yields fixed to expectations, we validate this assumption by allowing each fixed $B$ background yield to vary in the fit. We obtain background yields consistent with our expectations, and observe the shifts on signal parameters listed in Table IV. A systematic

TABLE IV. The shifts on signal yield [ $\delta N($ signal $)]$, fraction of longitudinally polarized events $\left(\delta f_{L}\right), S_{\text {long }}\left(\delta S_{\text {long }}\right)$, and $C_{\text {long }}$ ( $\delta C_{\text {long }}$ ) obtained when floating the yield of each fixed $B$ background in turn. The symbol $\dagger$ indicates a longitudinally polarized final state, and NR denotes a nonresonant final state. The yields of decay modes not listed in this table are allowed to vary in the nominal fit.

\begin{tabular}{l|rrrr}
\hline \hline Decay mode & $\delta N($ signal $)$ & \multicolumn{1}{c}{$\delta f_{L}$} & \multicolumn{1}{c}{$\delta S_{\text {long }}$} & $\delta C_{\text {long }}$ \\
\hline$B^{+} \rightarrow K^{+} \pi^{+} \pi^{-}$ & 3 & -0.002 & -0.002 & -0.003 \\
$B^{+} \rightarrow \pi^{+} \pi^{0} \pi^{0}$ & 11 & 0.001 & 0.002 & -0.001 \\
$B^{+} \rightarrow \pi^{0} \rho^{+}$ & 9 & 0.000 & 0.001 & -0.002 \\
$B^{+} \rightarrow \rho^{+} \rho^{0} \dagger$ & -8 & -0.001 & -0.002 & 0.000 \\
$B^{+} \rightarrow \rho^{+} \pi^{+} \pi^{-}$ & 1 & 0.000 & -0.001 & -0.001 \\
$B^{+} \rightarrow K_{2}^{*}(1770) \rho$ & -5 & 0.001 & -0.001 & -0.000 \\
$B^{+} \rightarrow K_{0}^{*} \pi$ & -13 & -0.001 & -0.002 & 0.001 \\
$B^{+} \rightarrow a_{1}^{+} \pi^{0}$ & 17 & 0.003 & 0.006 & -0.001 \\
$B^{+} \rightarrow \rho^{+} \pi^{0} \pi^{0}$ & -2 & 0.000 & 0.001 & -0.002 \\
$B^{+} \rightarrow a_{1}^{0}\left(\rho^{+} \pi^{-}\right) \rho^{+} \dagger$ & -34 & -0.006 & -0.028 & -0.003 \\
$B^{+} \rightarrow a_{1}^{0} \pi^{+}$ & 5 & 0.001 & 0.004 & -0.001 \\
$B^{+} \rightarrow \operatorname{charmless}$ & -3 & -0.001 & -0.002 & 0.000 \\
$B^{0} \rightarrow \pi^{+} \pi^{-} \pi^{0}(\mathrm{NR})$ & -4 & 0.000 & 0.000 & 0.000 \\
$B^{0} \rightarrow \rho^{ \pm} \pi^{\mp}$ & -5 & 0.000 & -0.001 & 0.000 \\
$B^{0} \rightarrow \pi^{0} \pi^{0} K_{S}^{0}$ & -25 & -0.003 & -0.009 & -0.001 \\
$B^{0} \rightarrow a_{1}^{ \pm}\left(\rho^{0} \pi^{ \pm}\right) \pi^{\mp}$ & 1 & -0.002 & -0.001 & -0.002 \\
$B^{0} \rightarrow K_{2}^{*}(1770) \rho$ & -31 & -0.003 & -0.009 & -0.003 \\
$B^{0} \rightarrow a_{1}^{ \pm}\left(\rho^{ \pm} \pi^{0}\right) \rho^{\mp} \dagger$ & -25 & -0.002 & -0.025 & -0.004 \\
$B^{0} \rightarrow a_{1}^{ \pm}\left(\rho^{0} \pi^{ \pm}\right) \rho^{\mp} \dagger$ & 11 & 0.000 & 0.006 & 0.000 \\
$B^{0} \rightarrow a_{1}^{ \pm}\left(\rho^{ \pm} \pi^{0}\right) \pi^{\mp}$ & 10 & 0.002 & 0.003 & -0.002 \\
$B^{0} \rightarrow \operatorname{charmless}$ & 1 & 0.000 & 0.000 & -0.001 \\
\hline \hline
\end{tabular}


uncertainty corresponding to the largest observed deviation is assigned on our results.

Once these checks have been completed, the fitting procedure is frozen and we extract the values of $S_{\text {long }}$ and $C_{\text {long }}$ by removing the offset.

\section{RESULTS}

\section{A. Fit results}

From the ML fit described above, we obtain the following results:

$$
\begin{aligned}
N(\text { signal }) & =729 \pm 60(\text { stat }) \\
f_{L} & =0.992 \pm 0.024(\text { stat }) \\
S_{\text {long }} & =-0.17 \pm 0.20(\text { stat }) \\
C_{\text {long }} & =0.01 \pm 0.15(\text { stat })
\end{aligned}
$$

after correction for a $+38.5 \pm 5.3$ event fit bias (see Sec. VIII), a -76 event bias from SCF on the signal yield (see Sec. IX B), and a correction for a $-0.016 \pm 0.002$ fit bias on $f_{L}$. The correlation between $S_{\text {long }}$ and $C_{\text {long }}$ is -0.035 . We discuss the origin of these fit biases in Sec. IX B. We calculate the branching fraction of this decay using $\mathcal{B}=N_{\text {signal }} /\left(\epsilon N_{b \bar{b}}\right)$, where $\epsilon$ is the efficiency for signal corresponding to the observed $f_{L}$, and $N_{B \text { pairs }}$ is the number of $B \bar{B}$ pairs analyzed. We obtain

$$
\mathcal{B}\left(B^{0} \rightarrow \rho^{+} \rho^{-}\right)=(25.5 \pm 2.1(\text { stat })) \times 10^{-6} .
$$

The $B^{0} \rightarrow \rho^{\mp} \pi^{ \pm} \pi^{0}$ background yield obtained from the fit is $9.2 \pm 53.6$ events. Figure 6 shows distributions of $m_{\mathrm{ES}}$, $\Delta E, \cos \theta_{i}$, and $m_{\pi^{ \pm}} \pi^{0}$ for the Lepton and Kaon I tagged events with a loose requirement on $\mathcal{N}$. Relative to the total
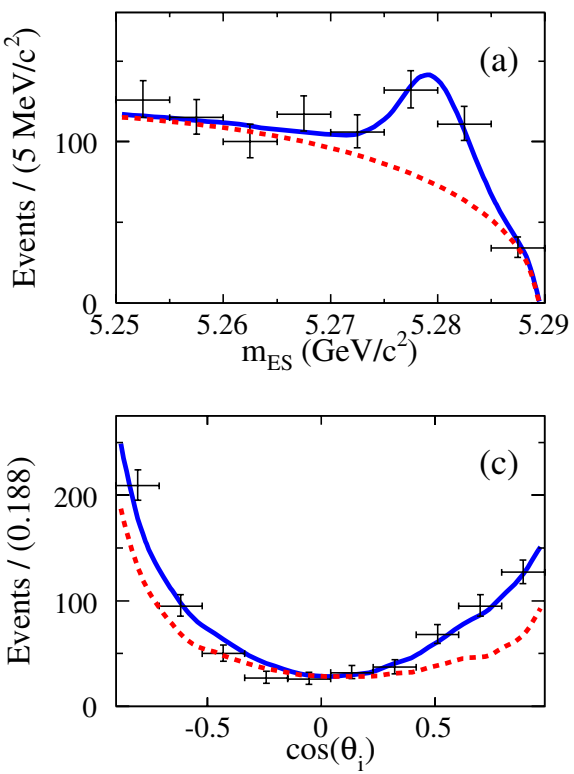

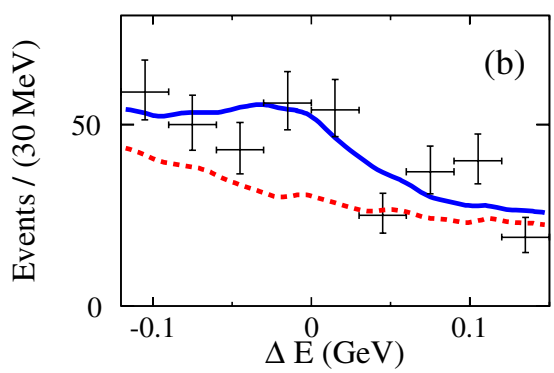

number of events in the fit, the plot of $m_{\mathrm{ES}}$ contains $15.6 \%$ of the signal and $1.1 \%$ of the total background. For the other plots there is an added constraint that $m_{\mathrm{ES}}>$ $5.27 \mathrm{GeV} / c^{2}$; these requirements retain $13.9 \%$ of the signal and $0.4 \%$ of the total background. Figure 7 shows the $\Delta t$ distribution for $B^{0}$ and $\bar{B}^{0}$ tagged events, as well as the time-dependent decay-rate asymmetry of Eq. (10). Here we apply the same selection criteria as in Figs. 6(b)-6(d).

\section{B. Systematic uncertainty studies}

Table V lists the possible sources of systematic uncertainties on the values of $N_{\text {signal }}, f_{L}, S_{\text {long }}$, and $C_{\text {long }}$ that have been studied, and are described in the following.

(i) The uncertainty from PDF parametrization is obtained by varying PDF shape parameters by $\pm 1 \sigma$, in turn. The deviations obtained are added in quadrature to give the quoted uncertainty.

(ii) The systematic uncertainty from the fraction of SCF events is obtained from the difference between the nominal fit result and that obtained when fitting the data and removing the SCF from the fit model. In the case of the signal yield we correct for half of the difference observed, and use $100 \%$ of the correction as an uncertainty. The uncertainty on the other signal observables comes from the difference observed between the two sets of fit results.

(iii) The kinematic endpoint position in $m_{\mathrm{ES}}$ is extracted from the fit. Changes in beam energy in the data can affect the endpoint position. To account for possible effects of this, we vary the kinematic endpoint position in $m_{\mathrm{ES}}$ by $\pm 0.45 \mathrm{MeV} / c^{2}$ which is determined from control samples of fully reconstructed $B^{0}$ decays.

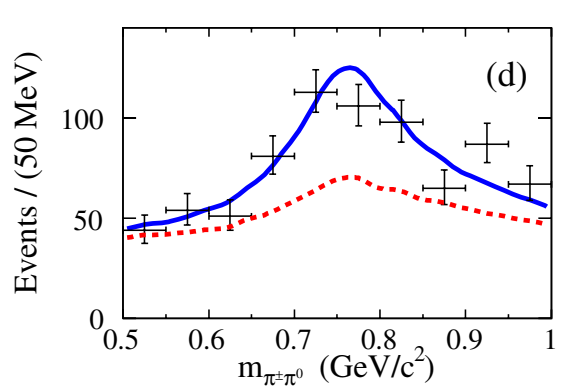

FIG. 6 (color online). The distributions for the highest purity tagged events for the variables (a) $m_{\mathrm{ES}}$, (b) $\Delta E$, (c) cosine of the $\rho$ helicity angle, and (d) $m_{\pi^{ \pm}} \pi^{0}$. The dashed lines are the sum of backgrounds, and the solid lines are the full PDF. 


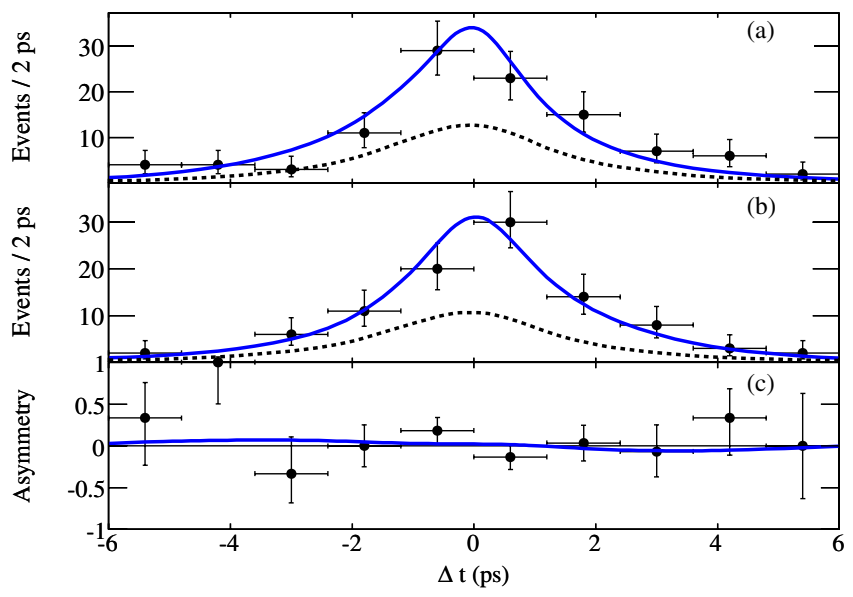

FIG. 7 (color online). The $\Delta t$ distribution for a sample of events enriched in signal for (a) $B^{0}$ and (b) $\bar{B}^{0}$ tagged events. The dashed lines are the sum of backgrounds, and the solid lines are the sum of signal and backgrounds. The time-dependent $C P$ asymmetry (see text) is shown in (c), where the curve is the measured asymmetry.

(iv) The uncertainty from the $m_{\mathrm{ES}}$ and $\Delta E$ widths is obtained from the observed shifts relative to our nominal result, when allowing these parameters to vary independently in the fit to data.

(v) We vary the $B$ background normalization within expectations for each background in turn. The deviations obtained are added in quadrature to give the quoted uncertainty from this source.

(vi) As the branching fractions of some of the $B$ backgrounds are not well known, we assign an additional uncertainty coming from the maximum shifts ob- tained when allowing each of the fixed backgrounds to vary, in turn, in the fit to data.

(vii) Additional uncertainties on the $C P$ results come from possible $C P$ violation in the $B$ background. We replace the $\Delta t$ PDFs used by each of the $B$ backgrounds, in turn, by one resembling the signal. Charged $B$ backgrounds can have nonzero values of $A_{C P}$, and neutral $B$ backgrounds can have nonzero values of $S$ and $C$. We use existing experimental constraints where possible; otherwise we allow for a $C P$ asymmetry up to $10 \%$ in $B$ decays to final states with charm, and up to $50 \%$ in $B$ decays to charmless final states.

(viii) The physics parameters $\tau_{B^{0}}=1.530 \pm 0.009 \mathrm{ps}$ and $\Delta m_{d}=0.507 \pm 0.005 \hbar / \mathrm{ps}$ [28] are varied within the quoted uncertainty.

(ix) The tagging efficiency and mistag fractions for signal and the $B$ backgrounds are corrected for data/MC differences observed in samples of fully reconstructed hadronic $B$ decays. Each of the tagging and mistag parameters is varied, in turn, by the uncertainty from the correction. The deviations obtained are added in quadrature to give the quoted uncertainty from this source.

(x) Allowing for possible $C P$ violation in the transverse polarization and in the WT longitudinally polarized signal SCF events results in additional uncertainties on signal observables. We vary $S$ and $C$ by \pm 0.5 ( \pm 1.0) for the transverse polarization (WT SCF).

(xi) Possible $C P$ violation from interference in doubly Cabibbo-suppressed decays (DCSD) on the tag side of the event [53] contributes to systematic uncertainties on $S_{\text {long }}$ and $C_{\text {long }}$.

TABLE V. Summary of additive systematic uncertainty contributions on the signal yield $N_{\text {signal }}, f_{L}, S_{\text {long }}$, and $C_{\text {long }}$.

\begin{tabular}{|c|c|c|c|c|}
\hline Contribution & $\sigma\left(N_{\text {signal }}\right)$ & $\sigma\left(f_{L}\right)$ & $\sigma\left(S_{\text {long }}\right)$ & $\sigma\left(C_{\text {long }}\right)$ \\
\hline PDF parametrization & $\begin{array}{l}+23 \\
-41\end{array}$ & ${ }_{-0.005}^{+0.019}$ & ${ }_{-0.04}^{+0.02}$ & 0.03 \\
\hline SCF fraction & 76 & 0.003 & 0.00 & 0.02 \\
\hline Control samples calibration & 16 & 0.006 & 0.01 & 0.01 \\
\hline$m_{\mathrm{ES}}$ endpoint & 12 & 0.001 & 0.00 & 0.01 \\
\hline$B$ background normalization & ${ }_{-20}^{+16}$ & ${ }_{-0.002}^{+0.005}$ & 0.01 & 0.00 \\
\hline Floating $B$ backgrounds & 34 & 0.006 & 0.03 & 0.00 \\
\hline$B$ background $C P$ asymmetry & 2 & ${ }_{-0.000}^{+0.001}$ & 0.00 & $\begin{array}{l}+0.02 \\
-0.01\end{array}$ \\
\hline$\tau_{B}$ & $\begin{array}{l}+0 \\
-1\end{array}$ & ${ }_{-0.000}^{+0.001}$ & 0.00 & 0.00 \\
\hline$\Delta m_{d}$ & $\begin{array}{l}+0 \\
{ }_{-1}^{+}\end{array}$ & ${ }_{-0.000}^{+0.001}$ & 0.00 & 0.00 \\
\hline Tagging and dilution & $\begin{array}{l}+2 \\
-16\end{array}$ & ${ }_{-0.001}^{+0.010}$ & 0.00 & 0.01 \\
\hline Transverse polarization $C P$ asymmetry & $\begin{array}{l}+0 \\
{ }_{-9}^{+0}\end{array}$ & ${ }_{-0.000}^{+0.006}$ & 0.01 & 0.01 \\
\hline Wrong track SCF $C P$ asymmetry & $\begin{array}{l}+0 \\
+3\end{array}$ & ${ }_{-0.000}^{+0.000}$ & 0.01 & 0.01 \\
\hline DCSD decays & $\cdots$ & $\cdots$ & 0.01 & 0.04 \\
\hline Interference & 18 & 0.000 & 0.01 & 0.01 \\
\hline Fit bias & 19 & 0.008 & 0.02 & 0.02 \\
\hline SVT alignment & $\cdots$ & $\cdots$ & 0.01 & 0.01 \\
\hline Total & $\begin{array}{l}+94 \\
-102\end{array}$ & ${ }_{-0.01}^{+0.03}$ & $\begin{array}{l}+0.05 \\
{ }_{-0.06}\end{array}$ & 0.06 \\
\hline
\end{tabular}


(xii) We estimate the systematic error on our results coming from neglecting the interference between $B^{0} \rightarrow$ $\rho^{+} \rho^{-}$and other $4 \pi$ final states: $B \rightarrow a_{1} \pi, \rho \pi \pi^{0}$, and $\pi \pi \pi^{0} \pi^{0}$. Details of this calculation are given in the Appendix.

(xiii) As the PDFs used in the ML fit do not account for all of the correlations between discriminating variables used in the fit, the results have a small bias. We calculate the fit bias on the signal observables as described in Sec. VIII. The observed bias on $N_{\text {signal }}$ and $f_{L}$ is corrected, and $100 \%$ of the correction is assigned as a systematic uncertainty. We do not observe a significant bias on $S_{\text {long }}$ and $C_{\text {long, }}$, and we conservatively assign a systematic uncertainty from fit bias based on the statistical precision of this test.

(xiv) Small imperfections in the knowledge of the geometry of the SVT over time can affect the measurement of $S_{\text {long }}$ and $C_{\text {long. }}$. We vary the alignment according to the results obtained from the study of $e^{+} e^{-} \rightarrow$ $e^{+} e^{-}, \mu^{+} \mu^{-}$events in order to estimate the magnitude of this systematic error on our $C P$ results.

The branching fraction has multiplicative systematic uncertainties from the reconstruction of $\pi^{0}$ mesons in the detector $(6 \%)$, uncertainties in the reconstruction of charged particles $(0.8 \%)$, and the discrimination of $\pi^{ \pm}$ from other types of charged particles $(1 \%)$. In addition to these uncertainties, there is a $1.1 \%$ uncertainty on the number of $B \bar{B}$ pairs in the data sample. The statistical uncertainties arising from the MC samples used in this analysis are negligible.

\section{CONSTRAINTS ON THE UNITARITY TRIANGLE}

\section{A. The $S U(2)$ isospin analysis}

In $S U(2)$ isospin symmetry, the amplitudes of $B$ decays to $\rho \rho$ final states [20] are related by

$$
\begin{aligned}
& \frac{1}{\sqrt{2}} A^{+-}=A^{+0}-A^{00}, \\
& \frac{1}{\sqrt{2}} \bar{A}^{+-}=\bar{A}^{-0}-\bar{A}^{00},
\end{aligned}
$$

for the longitudinal polarization and each of the $C P$ eigenstates of the transverse polarization, where $A^{i j}\left(\bar{A}^{i j}\right)$ are the amplitudes of $B(\bar{B})$ decays to the final state with charge $i j$, where $i=+,-, 0$ and $j=-, 0$. These two relations correspond to triangles in a complex plane as shown in Fig. 8. In the usual phase convention [20], the amplitudes $\bar{A}$ are rotated to $\tilde{A}$ in order to align the base of the triangles. After aligning $A^{+0}$ and $\bar{A}^{-0}$, the phase difference between $A^{+-}$ and $\bar{A}^{+-}$is $2 \delta \alpha$. There are two sources of ambiguities on the measurement of $\alpha$. There is a fourfold ambiguity coming from the orientation of the isospin triangles, and a twofold ambiguity from the trigonometric conversion in

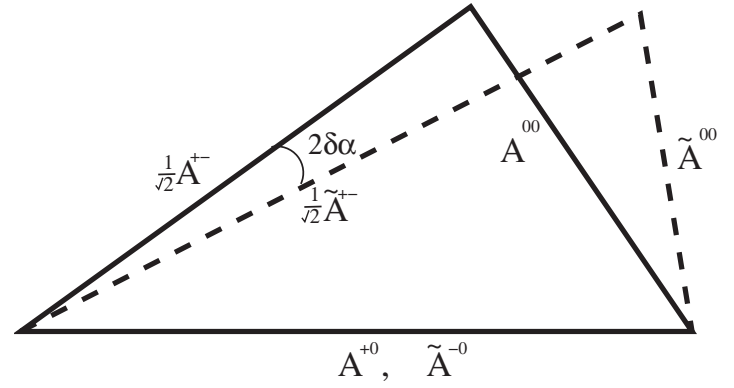

FIG. 8. The isospin triangle for $B \rightarrow \rho \rho$ decays.

Eq. (12). In order to measure $\alpha$, one must measure the branching fractions and charge asymmetries of $B$ decays to $\rho^{+} \rho^{-}, \rho^{ \pm} \rho^{0}, \rho^{0} \rho^{0}$.

There are several assumptions implicitly used in the isospin-based direct measurements of $\alpha$ :

(i) This approach only considers tree and gluonic penguin contributions. Possible contributions from electroweak penguins (EWP) are neglected as they do not obey $S U(2)$ isospin symmetry. These EWPs have the same topology as the gluonic penguin diagram in Fig. 2, with the gluon replaced by $\gamma$ or $Z^{0}$ bosons. In the absence of EWP contributions $\left|A^{+0}\right|=\left|\bar{A}^{-0}\right|$, and $A_{C P}$ is zero for $B^{+} \rightarrow \rho^{+} \rho^{0}$. Several groups have estimated the correction due to the $S U(2)$ breaking effect of EWP contributions to be 1.52.0 degrees $[54,55]$. These estimates consider contributions from the two EWP operators assumed to be dominant in the effective Hamiltonian.

(ii) The possible effect on the isospin analysis from $\rho^{0}-\omega$ mixing [55] is neglected.

(iii) Other $S U(2)$ symmetry breaking effects are neglected. Estimates of the magnitude of these effects are much less than the current experimental precision [56,57]. Possible isospin 5/2 amplitudes also break the $S U(2)$ triangle construction [58].

(iv) The isospin analysis outlined above neglects possible $I=1$ amplitudes [59]. The presence of $I=1$ amplitudes in $B^{0} \rightarrow \rho^{+} \rho^{-}$can be tested by measuring $S_{\text {long }}$ and $C_{\text {long }}$ for different ranges of the invariant $\pi^{ \pm} \pi^{0}$ mass.

We constrain the CKM angle $\alpha$ and penguin contribution $\delta \alpha$ from an isospin analysis of $B \rightarrow \rho \rho$ decays. The inputs to the isospin analysis are the amplitudes of the $C P$-even longitudinal polarization of the $\rho \rho$ final state, as well as the measured values of $S_{\text {long }}$ and $C_{\text {long }}$ for $B^{0} \rightarrow$ $\rho^{+} \rho^{-}$. We use the following numerical inputs in the isospin analysis:

(i) The measurements of $\mathcal{B}\left(B^{0} \rightarrow \rho^{+} \rho^{-}\right), f_{L}, S_{\text {long }}$, and $C_{\text {long }}$ presented here.

(ii) The measurements of $\mathcal{B}$ and $f_{L}$ for $B^{+} \rightarrow \rho^{+} \rho^{0}$ from Ref. [21].

(iii) The measurement of $\mathcal{B}\left(B^{0} \rightarrow \rho^{0} \rho^{0}\right)$ from Ref. [23].

To interpret our results in terms of a constraint on $\alpha$ from the isospin relations, we construct a $\chi^{2}$ that includes the 
measured quantities expressed as the lengths of the sides of the isospin triangles, and we determine the minimum $\chi_{0}^{2}$. We have adopted a simulated-experiment technique to compute the confidence level (CL) on $\alpha$; our method is similar to the approach proposed in Ref. [60]. For each value of $\alpha$, scanned between 0 and $180^{\circ}$, we determine the difference $\Delta \chi_{\text {DATA }}^{2}(\alpha)$ between the minimum of $\chi^{2}(\alpha)$ and $\chi_{0}^{2}$. We then generate MC experiments around the central values obtained from the fit to data with the given value of $\alpha$, and we apply the same procedure. The fraction of these experiments in which $\Delta \chi_{\mathrm{MC}}^{2}(\alpha)$ is smaller than $\Delta \chi_{\text {DATA }}^{2}(\alpha)$ is interpreted as the CL on $\alpha$. Figure 9 shows $1-\mathrm{CL}$ for $\alpha$ obtained from this method.

It is possible to obtain a prediction of $\alpha$ from indirect constraints by combining measurements of the CKM matrix elements $\left|V_{\mathrm{us}}\right|,\left|V_{\mathrm{ud}}\right|,\left|V_{\mathrm{ub}}\right|$, and $\left|V_{\mathrm{cb}}\right| ; C P$ violation in mixing from neutral kaons; $B-\bar{B}$ mixing in $B_{d}$ and $B_{s}$ mesons; and the measurement of $\sin 2 \beta$ from $b \rightarrow c \bar{c} s$ decays. The indirect constraint on $\alpha$ from the UTfit [15] and CKMfitter [14] groups are $(92.9 \pm 5.7)^{\circ}$ and $\left(100_{-7.3}^{+4.5}\right)^{\circ}$, respectively. Recent calculations using QCD factorization predict $\alpha=\left(85.6_{-7.3}^{+7.4}\right)^{\circ}$ [61]. Selecting the solution closest to the CKM combined fit average, we find $\alpha=[73.1,117.0]^{\circ}$ at $68 \% \mathrm{CL}$, where the error is dominated by $|\delta \alpha|$ which is $18^{\circ}$ at $68 \% \mathrm{CL}$. The constraint obtained on $\alpha$ is worse than the previous result in Ref. [26] for the following reasons: (i) the central value of the branching fraction for $B^{+} \rightarrow \rho^{+} \rho^{0}$ [21] is smaller than the value previously used [22,62], and (ii) the results of the latest search for $B^{0} \rightarrow \rho^{0} \rho^{0}$ show evidence for a signal [23], while the previous constraint on $\alpha$ used the central value corresponding to the upper limit available at that time [63]. Both of these factors lead to an increase in the penguin contribution to the total uncertainty on $\alpha$ when using an isospin analysis.

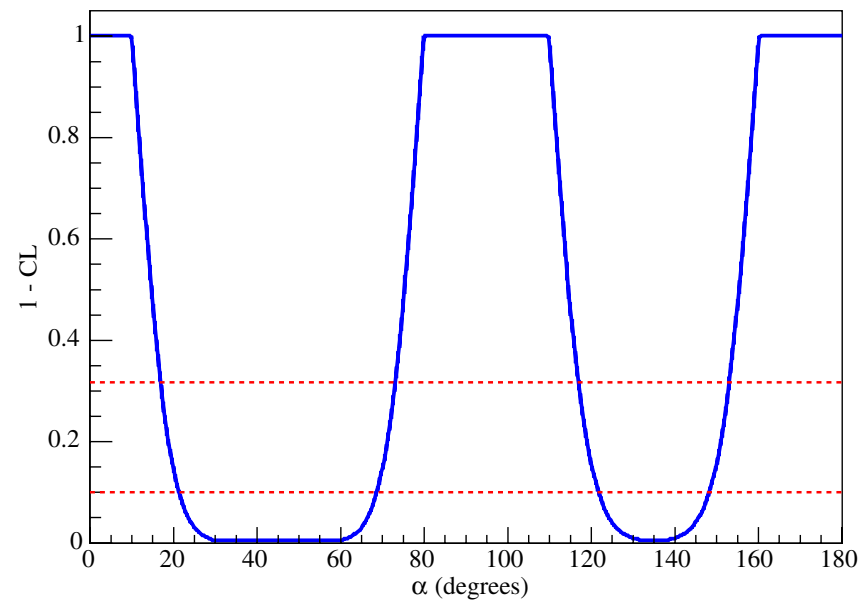

FIG. 9 (color online). Confidence level on $\alpha$ obtained from the isospin analysis with the statistical method described in [14]. The dashed lines correspond to the 68\% (top) and 90\% (bottom) CL intervals.
A future extension to the $S U(2)$ isospin method to measure $\alpha$ described here will be possible when there are sufficient statistics to perform a time-dependent analysis of $B^{0} \rightarrow \rho^{0} \rho^{0}$. The parameters $S_{\text {long }}$ and $C_{\text {long }}$ measured in $B^{0} \rightarrow \rho^{0} \rho^{0}$ can be incorporated into the isospin analysis, allowing one to overconstrain the isospin triangles of Eqs. (28) and (29).

\section{B. Flavor $S U(3)$ analysis}

There has been progress on constraining the penguin contribution in $B^{0} \rightarrow \rho^{+} \rho^{-}$using a flavor $S U(3)$-based approach and experimental constraints from $B^{+} \rightarrow$ $K^{* 0} \rho^{+}$[24]. The amplitude for $B^{0} \rightarrow \rho^{+} \rho^{-}$can be written as

$$
\mathcal{A}\left(B^{0} \rightarrow \rho^{+} \rho^{-}\right)=T e^{i \gamma}+P e^{i \delta_{T P}},
$$

where $T$ and $P$ are the magnitudes of tree and penguin amplitudes, $\gamma$ is the phase of $V_{\mathrm{ub}}$, and $\delta_{T P}$ is the strong phase difference between $T$ and $P$. The approach of Ref. [24] relates the penguin amplitude in $B^{0} \rightarrow \rho^{+} \rho^{-}$to the amplitude of the penguin decay $B^{+} \rightarrow K^{* 0} \rho^{+}$, giving three relations:

$$
\begin{gathered}
C_{\text {long }}=\frac{2 r \sin \delta_{T P} \sin (\beta+\alpha)}{1-2 r \cos \delta_{T P} \cos (\beta+\alpha)+r^{2}}, \\
S_{\text {long }}=\frac{\sin 2 \alpha+2 r \cos \delta_{T P} \sin (\beta-\alpha)-r^{2} \sin 2 \beta}{1-2 r \cos \delta_{T P} \cos (\beta+\alpha)+r^{2}}, \\
\left(\frac{\left|V_{\mathrm{cd}}\right| f_{\rho}}{\left|V_{\mathrm{cs}}\right| f_{K^{*}}}\right)^{2} \frac{\Gamma_{\mathrm{L}}\left(B^{ \pm} \rightarrow K^{* 0} \rho^{+}\right)}{\Gamma_{\mathrm{L}}\left(B^{0} \rightarrow \rho^{+} \rho^{-}\right)} \\
=\frac{F r^{2}}{1-2 r \cos \delta_{T P} \cos (\beta+\alpha)+r^{2}},
\end{gathered}
$$

with three unknowns, $\alpha, r$, and $\delta_{T P}$. The parameter $r=$ $|P / T|$, and $\beta$ is the phase of $V_{\mathrm{td}}$. We use the value of $\beta$ obtained from $b \rightarrow c \bar{c} s$ decays [7,8]. The $C P$ averaged decay rates of the longitudinal components of $B^{ \pm} \rightarrow$ $K^{* 0} \rho^{+}$and $B^{0} \rightarrow \rho^{+} \rho^{-}\left(\Gamma_{\mathrm{L}}\right)$ are related by the squared ratio of CKM matrix elements and decay constants $\left(f_{i}\right.$, where $\rho$ or $K^{*}$ ) as shown in Eq. (33). The factor $F$ accounts for additional sources of $S U(3)$ breaking not described by the decay constants.

The assumptions used in this approach are:

(i) The amplitude in the penguin dominated decay $B^{+} \rightarrow K^{* 0} \rho^{+}$is related to the penguin amplitude in $B^{0} \rightarrow \rho^{+} \rho^{-}$.

(ii) The dominant $S U(3)$ breaking correction accounted for by $F$ is the neglect of annihilation diagrams in the $B^{+} \rightarrow K^{* 0} \rho^{+}$decay. We use the same value of $F$ as Ref. [24]: $F=0.9 \pm 0.6$.

The result is a constraint on $\alpha$ with theoretical uncertainty from penguin contributions of $(+2,-5)^{\circ}$, which represents a stronger constraint on the unitarity triangle than provided by the isospin analysis constraint. Figure 10 


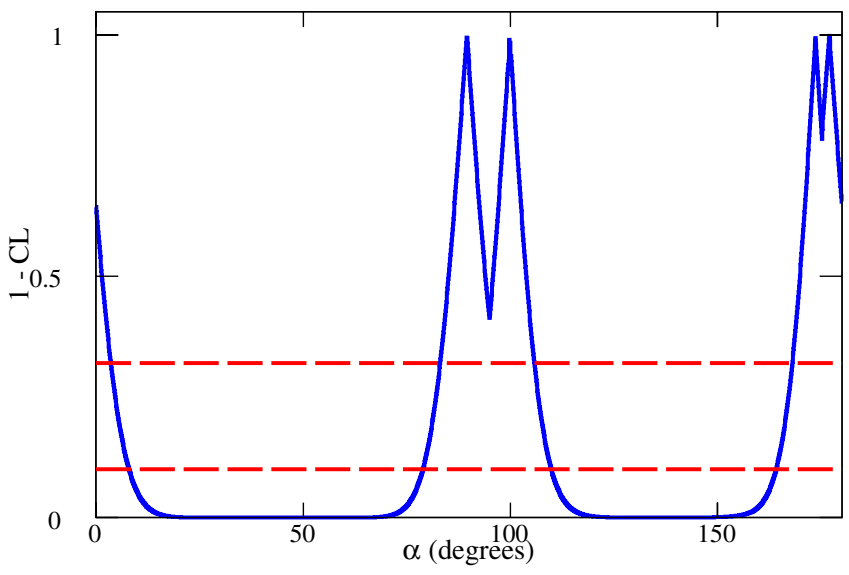

FIG. 10 (color online). Confidence level on $\alpha$ obtained from the flavor $S U(3)$ approach. The horizontal dashed lines correspond to the $68 \%$ (top) and $90 \%$ (bottom) CL intervals. The constraint shown in this figure uses some of the same inputs as the $S U(2)$ isospin constraint of Fig. 9, and the two cannot be averaged.

shows $1-\mathrm{CL}$ for $\alpha$ obtained with this method. Selecting the solution closest to the CKM combined fit average $[14,15]$, we find $\alpha=[83.3,105.8]^{\circ}$ at $68 \% \mathrm{CL}$, where the error is dominated by the experimental uncertainty on $S_{\text {long }}$ and $C_{\text {long }}$. The strong phase difference $\delta_{T P}$ is only weakly constrained, and $r=0.10_{-0.04}^{+0.03}$. The QCD factorization approach in Refs. [64-66] predicts that $\delta_{T P}$ is small, and so we require $\left|\delta_{T P}\right|<90^{\circ}$ as in Ref. [24]. We find $\alpha=\left(89.8_{-6.4}^{+7.0}\right)^{\circ}$. These constraints are in agreement with the prediction for $\alpha$ from UTfit [15] and CKMfitter [14]; the predictions are $(92.9 \pm 5.7)^{\circ}$ and $\left(100_{-7.3}^{+4.5}\right)^{\circ}$, respectively.

\section{CONCLUSIONS}

We report the measurement of the branching fraction, $f_{L}$, and $C P$ violation parameters, $S_{\text {long }}$ and $C_{\text {long }}$, for the decay $B^{0} \rightarrow \rho^{+} \rho^{-}$using a data sample of $(383.6 \pm 4.2) \times$ $10^{6} B \bar{B}$ pairs. We obtain the following results:

$$
\begin{aligned}
\mathcal{B}\left(B^{0} \rightarrow \rho^{+} \rho^{-}\right) & =\left(25.5 \pm 2.1(\text { stat })_{-3.9}^{+3.6}(\text { syst })\right) \times 10^{-6}, \\
f_{L} & =0.992 \pm 0.024(\text { stat })_{-0.013}^{+0.026}(\text { syst }), \\
S_{\text {long }} & =-0.17 \pm 0.20(\text { stat })_{-0.06}^{+0.05}(\text { syst }), \\
C_{\text {long }} & =0.01 \pm 0.15(\text { stat }) \pm 0.06(\text { syst }) .
\end{aligned}
$$

Using these results, and experimental knowledge of the other $B \rightarrow \rho \rho$ final states, we perform an isospin analysis to obtain a measurement of the CKM angle $\alpha$. The confidence level distribution for $\alpha$ is shown in Fig. 9. The solution obtained that is compatible with the results of SMbased fits of existing data is $\alpha=[73.1,117.0]^{\circ}$ at $68 \%$ CL. The uncertainty on the measurement of $\alpha$ from the isospin analysis is dominated by penguin pollution. If one uses the flavor $S U(3)$ approach described in the text to constrain $\alpha$, one obtains the constraint $\alpha=[83.3,105.8]^{\circ}$ at $68 \% \mathrm{CL}$.

\section{ACKNOWLEDGMENTS}

We are grateful for the extraordinary contributions of our PEP-II colleagues in achieving the excellent luminosity and machine conditions that have made this work possible. The success of this project also relies critically on the expertise and dedication of the computing organizations that support $B A B A R$. The collaborating institutions wish to thank SLAC for its support and the kind hospitality extended to them. This work is supported by the US Department of Energy and National Science Foundation, the Natural Sciences and Engineering Research Council (Canada), the Commissariat à l'Energie Atomique and Institut National de Physique Nucléaire et de Physique des Particules (France), the Bundesministerium für Bildung und Forschung and Deutsche Forschungsgemeinschaft (Germany), the Istituto Nazionale di Fisica Nucleare (Italy), the Foundation for Fundamental Research on Matter (The Netherlands), the Research Council of Norway, the Ministry of Science and Technology of the Russian Federation, Ministerio de Educación y Ciencia (Spain), and the Science and Technology Facilities Council (United Kingdom). Individuals have received support from the Marie-Curie IEF program (European Union) and the A. P. Sloan Foundation.

\section{APPENDIX: INTERFERENCE OF $B^{0} \rightarrow \rho^{+} \rho^{-}$ WITH OTHER $B^{0} \rightarrow 4 \pi$ MODES}

An extensive study of the $B$ backgrounds, and the associated systematic error, has been presented earlier in this paper. A number of $B$ backgrounds decay into the same final state as the signal. These are

(i) $B^{0} \rightarrow a_{1}^{ \pm} \pi^{\mp}, a_{1}^{ \pm} \rightarrow \rho^{ \pm} \pi^{0}$,

(ii) $B^{0} \rightarrow \rho^{\mp} \pi^{ \pm} \pi^{0}$, nonresonant,

(iii) $B^{0} \rightarrow \pi^{+} \pi^{-} \pi^{0} \pi^{0}$, nonresonant.

The systematic error associated with these modes was evaluated by propagating the uncertainty on the branching ratio and $C P$ asymmetries to the final measurements, with the appropriate description of acceptance, SCF, resolutions, and other reconstruction biases (see Sec. IX B). The likelihood described in Sec. VII does not account for possible interference between $B^{0} \rightarrow \rho^{+} \rho^{-}$and other $B^{0} \rightarrow 4 \pi$ final states.

Acceptance, SCF, and other reconstruction biases are not taken into account in this study, and instead, the $\rho^{+} \rho^{-}$ measurements are averaged in a perfect region of the phase space referred to as the " $\rho^{+} \rho^{-}$band," on which the analysis technique is assumed to have a uniform sensitivity. This $\rho^{+} \rho^{-}$band corresponds to the kinematic selection of $\rho^{ \pm}$(see Sec. III B): $0.5<m_{\pi^{ \pm} \pi^{0}}<1.0 \mathrm{GeV} / c^{2}$ and $-0.9<\cos \theta_{i}<0.98$.

The remainder of this appendix describes the final state wave function, decay amplitudes, effective $C P$ asymmetries, and the estimate of the systematic uncertainty from neglecting interference between the signal and other $B^{0} \rightarrow$ $4 \pi$ final states. 


\section{The final state wave functions}

This section summarizes the kinematic dependence of the decay amplitudes.

\section{a. Lorentz invariant phase space}

The four-particle final state can be described completely in terms of the following five variables:

(i) $m_{1}$ and $m_{2}$ : the masses of the $\pi^{+} \pi^{0}$ and $\pi^{-} \pi^{0}$ pairs.

(ii) $\cos \theta_{1}$ and $\cos \theta_{2}$ : the angle between the $\pi^{ \pm} \pi^{0}$ pair line of flight (as seen in the $B$ frame) and the $\pi^{0}$ in the pion pair frame.

(iii) $\phi$ : the angle between the two planes defined by the $\pi^{+} \pi^{0}$ and $\pi^{-} \pi^{0}$ pairs in the $B$ frame.

We write each phase-space dependent quantity $d \Phi_{4}$ as a function of these five variables. These are

$$
d \Phi_{4} \propto \frac{P_{B}^{\pi^{+}} \pi^{0}}{M_{B}} \frac{P_{\pi^{+} \pi^{0}}^{\pi^{0}}}{m_{1}} \frac{P_{\pi^{-}}^{\pi^{0}} \pi^{0}}{m_{2}} d m_{1}^{2} d m_{2}^{2} d \cos \theta_{1} d \cos \theta_{2} d \phi,
$$

where $P_{B}^{\pi^{+}} \pi^{0}$ denotes the momentum of the pion pair in the $B$ frame and $M_{B}$ is the mass of the $B$ (and similarly for the other quantities in this expression).

\section{b. Kinematic dependence of $\rho^{+} \rho^{-}$}

We assume that the longitudinal polarization of $\rho^{+} \rho^{-}$is dominant, so the kinematic dependence of the $\rho^{+} \rho^{-}$wave function is

$$
\begin{aligned}
f\left(\rho^{+} \rho^{-}\right) & \propto \mathrm{BW}\left(m_{1}\right) \mathrm{BW}\left(m_{2}\right) Y_{1}^{0}\left(\theta_{1}\right) Y_{1}^{0}\left(\theta_{2}\right) \\
& \propto \mathrm{BW}\left(m_{1}\right) \mathrm{BW}\left(m_{2}\right) \cos \left(\theta_{1}\right) \cos \left(\theta_{2}\right),
\end{aligned}
$$

where BW denotes a Breit-Wigner function (the simple nonrelativistic form is assumed), and $Y_{l}^{m}$ are the spherical harmonics.

\section{c. Kinematic dependence of $a_{1} \pi$}

In order to write the wave function of $a_{1}^{+} \pi^{-}$, we need to define the following three variables, which are functions of the variables found in Appendix A 1 a:

(i) $\theta_{\rho}$ : the angle between the $\pi^{0}$ and the $\rho$ line of flight (as seen from the $a_{1}$ ) in the $\rho$ frame.

(ii) $\theta_{a_{1}}$ : the angle between the $\rho$ and $a_{1}$ line of flight (as seen from the $B$ ) in the $a_{1}$ frame.

(iii) $\phi_{a_{1}}$ : the angle between the two decay planes of the $a_{1}$ and $\rho$ mesons in the $a_{1}$ frame.

A schematic view of $B^{0} \rightarrow a_{1}^{ \pm} \pi^{\mp}$ showing $\theta_{\rho}$ and $\theta_{a_{1}}$ is given in Fig. 11.

The $a_{1}$ has the possibility to decay both into an $S$ wave and a $D$ wave. We assume that the $S$ wave contribution is dominant, so the kinematic dependence of $a_{1} \pi$ is

$$
\begin{aligned}
f\left(a_{1} \pi\right) \propto & \operatorname{BW}\left(a_{1}\right) \operatorname{BW}(\rho)\left\{d_{0,1}^{1}\left(\theta_{a_{1}}\right) Y_{1}^{1}\left(\theta_{\rho}, \phi_{a_{1}}\right)\right. \\
& \left.+d_{0,0}^{1}\left(\theta_{a_{1}}\right) Y_{1}^{0}\left(\theta_{\rho}\right)+d_{0,-1}^{1}\left(\theta_{a_{1}}\right) Y_{1}^{-1}\left(\theta_{\rho}, \phi_{a_{1}}\right)\right\},
\end{aligned}
$$

where the $d_{m, m^{\prime}}^{j}$ are rotation matrices. A nonrelativistic Breit-Wigner function is assumed for $\operatorname{BW}(\rho)$, and a relativistic form is used for $\mathrm{BW}\left(a_{1}\right)$.

\section{d. Kinematic dependence of the nonresonant contributions}

The kinematic dependence of the nonresonant contributions can be written as

$$
f\left(\rho^{\mp} \pi^{ \pm} \pi^{0}\right) \propto \mathrm{BW}\left(\rho_{1}\right) Y_{1}^{0}\left(\theta_{1}\right),
$$

for $\rho^{\mp} \pi^{ \pm} \pi^{0}$, and

$$
f\left(\pi^{+} \pi^{-} \pi^{0} \pi^{0}\right)=\text { constant, }
$$

for the nonresonant four-pion final state.

We note that the spherical harmonics entering the $\rho^{+} \rho^{-}$ amplitudes found in Eq. (A2) differ from those found in these two amplitudes: this means that these wave functions are orthogonal, and after the integration over the helicity angles, the interfering terms $f\left(\rho^{+} \rho^{-}\right) f^{\star}\left(\rho^{\mp} \pi^{ \pm} \pi^{0}\right)$ and $f\left(\rho^{+} \rho^{-}\right) f^{\star}\left(\pi^{+} \pi^{-} \pi^{0} \pi^{0}\right)$ will cancel completely if the integration is done over the whole phase space. The " $\rho^{+} \rho^{-}$band" used in this analysis is slightly asymmetric in helicity angle, and as a result we can expect a small deviation from zero for these integrals.

\section{e. Symmetrization of the wave functions}

The final state is made of four bosons, two of which are identical (the two $\pi^{0}$ ). Thus each of the above wave functions needs to be symmetrized, that is to say that the amplitude

$$
f\left(p_{\pi^{+}}, p_{\pi^{0}}^{1}, p_{\pi^{-}}, p_{\pi^{0}}^{2}\right),
$$

where $p$ denotes the four-momentum of one of the pions, needs to be replaced by

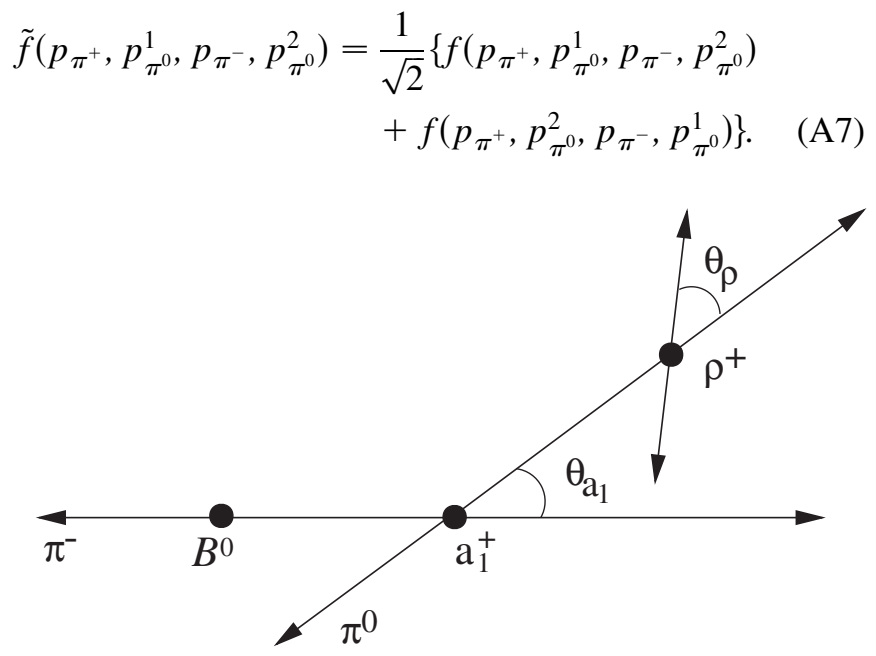

FIG. 11. Definition of the variables $\theta_{\rho}$ and $\theta_{a_{1}}$ entering the $a_{1} \pi$ kinematic dependence. 
In the rest of this study, each of the wave functions is symmetrized by $\pi^{0}$ meson exchange.

\section{Decay amplitudes}

For a particular contribution $B^{0} \rightarrow x$, the total decay amplitude $\mathcal{A}\left(B^{0} \rightarrow x\right)$ is the product of a phase-space independent amplitude $A\left(B^{0} \rightarrow x\right)$ and one of the above kinematic dependences $\tilde{f}(x)$ :

$$
\mathcal{A}\left(B^{0} \rightarrow x\right)=A\left(B^{0} \rightarrow x\right) \tilde{f}(x) .
$$

This section describes how we calculate $A\left(B^{0} \rightarrow x\right)$.

\section{a. Amplitude of $B \rightarrow \rho^{+} \rho^{-}$}

For $B \rightarrow \rho^{+} \rho^{-}$, two amplitudes need to be known: $A\left(B^{0} \rightarrow \rho^{+} \rho^{-}\right)$and $A\left(\bar{B}^{0} \rightarrow \rho^{+} \rho^{-}\right)$, corresponding to 4 degrees of freedom. We choose the following parametrization:

(i) The branching ratio $\mathcal{B}\left(B \rightarrow \rho^{+} \rho^{-}\right)$,

(ii) The $C P$-violating asymmetries $C_{\text {long }}\left(\rho^{+} \rho^{-}\right)$and $S_{\text {long }}\left(\rho^{+} \rho^{-}\right)$,

(iii) An overall phase $\delta\left(\rho^{+} \rho^{-}\right)$which may be set to zero in the appropriate phase convention.

The parameters $\mathcal{B}\left(B \rightarrow \rho^{+} \rho^{-}\right), \quad C_{\text {long }}\left(\rho^{+} \rho^{-}\right), \quad$ and $S_{\text {long }}\left(\rho^{+} \rho^{-}\right)$are set to the values reported here (see Sec. XI).

\section{b. Amplitude of $B \rightarrow a_{1} \pi$}

Four amplitudes describe the $B \rightarrow a_{1} \pi$ contributions, corresponding to a total of 8 degrees of freedom: $A\left(B^{0} \rightarrow\right.$ $\left.a_{1}^{+} \pi^{-}\right), \quad A\left(B^{0} \rightarrow a_{1}^{-} \pi^{+}\right), A\left(\bar{B}^{0} \rightarrow a_{1}^{+} \pi^{-}\right)$, and $A\left(\bar{B}^{0} \rightarrow\right.$ $\left.a_{1}^{-} \pi^{+}\right)$. We choose a parametrization similar to that of the time-dependent $a_{1} \pi$ analysis [67], for which parameters have direct physical meaning:

(i) $\mathcal{B}\left(B^{0} \rightarrow a_{1} \pi\right)$ is the total branching ratio.

(ii) $C\left(a_{1} \pi\right)+\Delta C\left(a_{1} \pi\right)=C^{+}\left(a_{1} \pi\right)=\frac{1-\left|\lambda_{C P}\left(a_{1}^{+} \pi^{-}\right)\right|^{2}}{1+\left|\lambda_{C P}\left(a_{1}^{+} \pi^{-}\right)\right|^{2}}$ where $\lambda_{C P}\left(a_{1}^{+} \pi^{-}\right)=\frac{q}{p} \frac{A\left(\bar{B}^{0} \rightarrow a_{1}^{+} \pi^{-}\right)}{A\left(B^{0} \rightarrow a_{1}^{+} \pi^{-}\right)}$.

(iii) $C\left(a_{1} \pi\right)-\Delta C\left(a_{1} \pi\right)=C^{-}\left(a_{1} \pi\right)=\frac{1-\left|\lambda_{C P}\left(a_{1}^{-} \pi^{+}\right)\right|^{2}}{1+\left|\lambda_{C P}\left(a_{1}^{-} \pi^{+}\right)\right|^{2}}$ where $\lambda_{C P}\left(a_{1}^{-} \pi^{+}\right)=\frac{q}{p} \frac{A\left(\bar{B}^{0} \rightarrow a_{1}^{-} \pi^{+}\right)}{A\left(B^{0} \rightarrow a_{1}^{-} \pi^{+}\right)}, C\left(a_{1} \pi\right)$ is a direct $C P$-violating asymmetry, and $\Delta C\left(a_{1} \pi\right)$ is $C P$ conserving.

(iv) $S\left(a_{1} \pi\right)+\Delta S\left(a_{1} \pi\right)=S^{+}\left(a_{1} \pi\right)=\frac{2 \operatorname{Im}\left\{\lambda_{C P}\left(a_{1}^{+} \pi^{-}\right)\right\}}{1+\left|\lambda_{C P}\left(a_{1}^{+} \pi^{-}\right)\right|^{2}}$.

(v) $S\left(a_{1} \pi\right)-\Delta S\left(a_{1} \pi\right)=S^{-}\left(a_{1} \pi\right)=\frac{2 \operatorname{Im}\left\{\lambda_{C P}\left(a_{1}^{-} \pi^{+}\right)\right\}}{1+\left|\lambda_{C P}\left(a_{1}^{-} \pi^{+}\right)\right|^{2}}$, where $S\left(a_{1} \pi\right)$ is a $C P$-violating asymmetry in the interference between decay and mixing, and $\Delta S\left(a_{1} \pi\right)$ is $C P$ conserving.

(vi) $A_{C P}\left(a_{1} \pi\right)=\frac{\left|A^{+-}\right|^{2}+\left|\bar{A}^{+-}\right|^{2}-\left|A^{-+}\right|^{2}-\left|\bar{A}^{-+}\right|^{2}}{\left|A^{+-}\right|^{2}+\left|\bar{A}^{+-}\right|^{2}-\left|A^{-+}\right|^{2}-\left|\bar{A}^{-+}\right|^{2}}$ where $A^{ \pm \mp}=$ $A\left(B^{0} \rightarrow a_{1}^{ \pm} \pi^{\mp}\right)$, and $\bar{A}^{ \pm \mp}$ is its complex conjugate. These are also direct $C P$ violating asymmetries.

(vii) Two overall phases of the final states $\delta\left(a_{1}^{+} \pi^{-}\right)$and $\delta\left(a_{1}^{-} \pi^{+}\right)$.

For $a_{1} \pi$ we assume that all of these parameters are constant over phase space.

\section{Effective $\boldsymbol{C P}$ asymmetries}

Considering the two final states $\rho^{+} \rho^{-}$and $a_{1} \pi$, the phase-space dependent four-pion decay amplitude is

$$
\begin{aligned}
\mathcal{A}\left(B^{0} \rightarrow 4 \pi\right)= & \mathcal{A}\left(B^{0} \rightarrow \rho^{+} \rho^{-}\right)+\mathcal{A}\left(B^{0} \rightarrow a_{1}^{+} \pi^{-}\right) \\
& +\mathcal{A}\left(B^{0} \rightarrow a_{1}^{-} \pi^{+}\right), \\
= & A\left(B^{0} \rightarrow \rho^{+} \rho^{-}\right) f\left(\rho^{+} \rho^{-}\right) \\
& +A\left(B^{0} \rightarrow a_{1}^{+} \pi^{-}\right) f\left(a_{1}^{+} \pi^{-}\right) \\
& +A\left(B^{0} \rightarrow a_{1}^{-} \pi^{+}\right) f\left(a_{1}^{-} \pi^{+}\right),
\end{aligned}
$$

with a similar expression for $\mathcal{A}\left(\bar{B}^{0} \rightarrow 4 \pi\right)$. Similar equations exist for $\rho \pi \pi$ and $4 \pi$ final states.

We can define the phase-space dependent quantity

$$
\lambda_{4 \pi}\left(\Phi_{4}\right)=\frac{q}{p} \frac{\mathcal{A}\left(\bar{B}^{0} \rightarrow 4 \pi\right)}{\mathcal{A}\left(B^{0} \rightarrow 4 \pi\right)},
$$

where $\lambda_{4 \pi}\left(\Phi_{4}\right)$ is a function of $m_{1}, m_{2}, \cos \theta_{1}, \cos \theta_{2}$, and $\phi$. Thus, we define the phase-space dependent $C P$-violating asymmetries

$$
\begin{aligned}
& C_{4 \pi}\left(\Phi_{4}\right)=\frac{1-\left|\lambda_{4 \pi}\left(\Phi_{4}\right)\right|^{2}}{1+\left|\lambda_{4 \pi}\left(\Phi_{4}\right)\right|^{2}}, \\
& S_{4 \pi}\left(\Phi_{4}\right)=\frac{2 \operatorname{Im}\left(\lambda_{4 \pi}\left(\Phi_{4}\right)\right)}{1+\left|\lambda_{4 \pi}\left(\Phi_{4}\right)\right|^{2}},
\end{aligned}
$$

that are analogous to Eqs. (8) and (9).

In principle, it is possible to measure the values of $C_{4 \pi}\left(\Phi_{4}\right)$ and $S_{4 \pi}\left(\Phi_{4}\right)$ at each point of phase space; however this would be experimentally challenging. Instead, this measurement uses integrated values $C_{\text {eff }}$ and $S_{\text {eff }}$, which can be seen as the averages of $C$ and $S$ across the previously defined $\rho^{+} \rho^{-}$band, weighted over the number of events found at each location of the phase space which corresponds to

$$
\begin{aligned}
C_{\text {eff }} & =\frac{\int_{\rho \rho \text { band }} C_{4 \pi}\left(\Phi_{4}\right)\left(\left|\mathcal{A}\left(B^{0} \rightarrow 4 \pi\right)\right|^{2}+\left|\mathcal{A}\left(\bar{B}^{0} \rightarrow 4 \pi\right)\right|^{2}\right) d \Phi_{4}}{\int_{\rho \rho \text { band }}\left(\left|\mathcal{A}\left(B^{0} \rightarrow 4 \pi\right)\right|^{2}+\left|\mathcal{A}\left(\bar{B}^{0} \rightarrow 4 \pi\right)\right|^{2}\right) d \Phi_{4}}, \\
S_{\text {eff }} & =\frac{\int_{\rho \rho \text { band }} S_{4 \pi}\left(\Phi_{4}\right)\left(\left|\mathcal{A}\left(B^{0} \rightarrow 4 \pi\right)\right|^{2}+\left|\mathcal{A}\left(\bar{B}^{0} \rightarrow 4 \pi\right)\right|^{2}\right) d \Phi_{4}}{\int_{\rho \rho \text { band }}\left(\left|\mathcal{A}\left(B^{0} \rightarrow 4 \pi\right)\right|^{2}+\left|\mathcal{A}\left(\bar{B}^{0} \rightarrow 4 \pi\right)\right|^{2}\right) d \Phi_{4}}
\end{aligned}
$$


We can also define the values of $C$ and $S$ that would be observed in the absence of interference effects, denoted by $C_{4 \pi}^{X}\left(\Phi_{4}\right)$ and $S_{4 \pi}^{X}\left(\Phi_{4}\right)$, and similarly the integrated values across the $\rho^{+} \rho^{-}$band, $C_{\text {eff }}^{\chi}$ and $S_{\text {eff }}^{X}$. Finally, for the evaluation of the systematic error, we are really interested in the biases on $C$ and $S$ purely due to interference, which are the two differences $\delta C=C_{\text {eff }}-C_{\text {eff }}^{x}$ and $\delta S=S_{\text {eff }}-$ $S_{\text {eff. }}^{\mathrm{X}}$. We adopt the same strategy for the branching fraction $\mathcal{B}\left(\rho^{+} \rho^{-}\right)$by defining $\delta \mathcal{B}=\mathcal{B}_{\text {eff }}-\mathcal{B}_{\text {eff }}^{X}$ in a similar way.

\section{The systematic error}

As the 8 parameters describing the amplitude $A\left(B^{0}\left(\bar{B}^{0}\right) \rightarrow a_{1} \pi\right)$ are not yet measured, to estimate the systematic error on $\rho^{+} \rho^{-}$parameters, we need to propagate the uncertainty on these 8 parameters. For this, we use a $\mathrm{MC}$ technique. For each experiment, we generate the $C P$ parameters $\quad C\left(a_{1} \pi\right), \quad \Delta C\left(a_{1} \pi\right), \quad S\left(a_{1} \pi\right), \quad \Delta S\left(a_{1} \pi\right)$, $A_{C P}\left(a_{1} \pi\right)$, the phases $\delta\left(a_{1}^{+} \pi^{-}\right)$and $\delta\left(a_{1}^{-} \pi^{+}\right)$, and the branching fraction $\mathcal{B}\left(a_{1} \pi\right)$. The values used to generate the $a_{1} \pi$ contribution have Gaussian PDFs for measured quantities (with mean and width corresponding to the measurement) and uniform PDFs for unmeasured quantities (sampled over the allowed values). Then, we estimate the value of $\delta C$ and $\delta S$ for each generated value of the $a_{1} \pi$ parameters. The systematic errors are taken as the RMS of the $\delta C, \delta S$, and $\delta \mathcal{B}$ distributions.

For this study, the quantity $\mathcal{B}\left(a_{1} \pi\right)$ is fixed to the value measured in $[51], \quad \mathcal{B}\left(B^{0} \rightarrow a_{1}^{+} \pi^{-}, a_{1}^{+} \rightarrow \rho^{+} \pi^{0}\right)=$
TABLE VI. Systematic error coming from interference effects due to three contaminating amplitudes.

\begin{tabular}{l|ccc}
\hline \hline Contribution & $\sigma(C)$ & $\sigma(S)$ & $\frac{\sigma(\mathcal{B})}{\mathcal{B}}$ \\
\hline$a_{1} \pi$ & 0.009 & 0.009 & $2.5 \%$ \\
$\rho \pi \pi$ nonresonant & 0.010 & 0.010 & $1.1 \%$ \\
$4 \pi$ nonresonant & 0.002 & 0.002 & $0.3 \%$ \\
Total & 0.014 & 0.014 & $2.7 \%$ \\
\hline \hline
\end{tabular}

$(16.6 \pm 2.4) \times 10^{-6}$, and the $C P$ parameters are obtained from [67]: $C\left(a_{1} \pi\right)=-0.10 \pm 0.17, \Delta C\left(a_{1} \pi\right)=0.26 \pm$ $0.17, \quad S\left(a_{1} \pi\right)=0.37 \pm 22, \quad \Delta S\left(a_{1} \pi\right)=-0.14 \pm 0.22$, and $A_{C P}\left(a_{1} \pi\right)=-0.07 \pm 0.07$.

We estimate the effect of interference between signal and either $\rho^{\mp} \pi^{ \pm} \pi^{0}$ or $\pi^{+} \pi^{-} \pi^{0} \pi^{0}$ in a similar way, where we assume the branching fraction for these two nonresonant backgrounds is $(5.7 \pm 13.0) \times 10^{-6}$. This value is obtained by modeling the $\rho^{\bar{\mp}} \pi^{ \pm} \pi^{0}$ component in the likelihood fit and by fitting a free yield to the data, and we assume that $\rho^{\mp} \pi^{ \pm} \pi^{0}$ and $\pi^{+} \pi^{-} \pi^{0} \pi^{0}$ have the same branching fraction.

We summarize the systematic uncertainty obtained for the different $4 \pi$ final states considered here in Table VI. The total uncertainty on $\mathcal{B}$ is used to calculate the quoted systematic uncertainty on $f_{L}$ from this source. We conclude that interference between signal and other $4 \pi$ final states has a negligible impact on the measurement.
[1] J. H. Christenson, J. W. Cronin, V. L. Fitch, and R. Turlay, Phys. Rev. Lett. 13, 138 (1964).

[2] A. D. Sakharov, Pis'ma Zh. Eksp. Teor. Fiz. 5, 32 (1967) [JETP Lett. 5, 24 (1967)].

[3] N. Cabibbo, Phys. Rev. Lett. 10, 531 (1963).

[4] M. Kobayashi and T. Maskawa, Prog. Theor. Phys. 49, 652 (1973).

[5] L. Wolfenstein, Phys. Rev. Lett. 51, 1945 (1983).

[6] A. J. Buras, M.E. Lautenbacher, and G. Ostermaier, Phys. Rev. D 50, 3433 (1994).

[7] B. Aubert et al. (BABAR Collaboration), Phys. Rev. Lett. 89, 201802 (2002).

[8] K. Abe et al. (Belle Collaboration), Phys. Rev. D 66, 071102 (2002).

[9] M. B. Gavela, Mod. Phys. Lett. A 9, 795 (1994).

[10] P. Huet and E. Sather, Phys. Rev. D 51, 379 (1995).

[11] B. Aubert et al. (BABAR Collaboration), arXiv:hep-ex/ 0703021.

[12] K.-F. Chen et al. (Belle Collaboration), Phys. Rev. Lett. 98, 031802 (2007).

[13] M. Ciuchini, E. Franco, G. Martinelli, L. Reina, and L. Silvestrini, Z. Phys. C 68, 239 (1995).

[14] J. Charles et al. (CKMfitter Group), Eur. Phys. J. C 41, 1
(2005). Updated results and plots are available at http:// ckmfitter.in2p3.fr.

[15] M. Bona et al. (UTfit Collaboration), J. High Energy Phys. 07 (2005) 028. Updated results and plots are available at http://www.utfit.org.

[16] I. Bigi and A. Sanda, CP Violation (Cambridge University Press, Cambridge, 1999).

[17] G. Branco, L. Lavoura, and J. Silva, CP Violation (Oxford University Press, New York, 1999).

[18] Unless explicitly stated, charge conjugation is implied throughout.

[19] A. Bevan, Mod. Phys. Lett. A 21, 305 (2006).

[20] M. Gronau and D. London, Phys. Rev. Lett. 65, 3381 (1990).

[21] B. Aubert et al. (BABAR Collaboration), Phys. Rev. Lett. 97, 261801 (2006).

[22] J. Zhang et al. (Belle Collaboration), Phys. Rev. Lett. 91, 221801 (2003).

[23] B. Aubert et al. (BABAR Collaboration), Phys. Rev. Lett. 98, 111801 (2007).

[24] M. Beneke, M. Gronau, J. Rohrer, and M. Spranger, Phys. Lett. B 638, 68 (2006).

[25] See, for example, J. Zupan, arXiv:hep-ph/0701004, and 
references therein.

[26] B. Aubert et al. (BABAR Collaboration), Phys. Rev. Lett. 95, 041805 (2005).

[27] A. Somov et al. (Belle Collaboration), Phys. Rev. Lett. 96, 171801 (2006).

[28] W.-M. Yao et al. (Particle Data Group), J. Phys. G 33, 1 (2006).

[29] See, for example, D. Kirkby and Y. Nir, Sec. 12, in Ref. [28].

[30] B. Aubert et al. (BABAR Collaboration), Phys. Rev. Lett. 93, 231801 (2004).

[31] A. Somov et al. (Belle Collaboration), Phys. Rev. D 76, 011104 (2007).

[32] PEP-II Conceptual Design Report No. SLAC-R-418, 1993.

[33] B. Aubert et al. (BABAR Collaboration), Nucl. Instrum. Methods Phys. Res., Sect. A 479, 1 (2002).

[34] G. Benelli, K. Honscheid, E. A. Lewis, J. J. Regensburger, and D. S. Smith, Nuclear Science Symposium Conference Record, 2005 (IEEE, New York, 2005), Vol. 2, p. 1145.

[35] W. Menges, Nuclear Science Symposium Conference Record, 2005 (IEEE, New York, 2005), Vol. 3, p. 1470.

[36] M. R. Convery, P. C. Kim, H. P. Paar, C. H. Rogers, R. H. Schindler, S. K. Swain, and C. C. Young, Nucl. Instrum. Methods Phys. Res., Sect. A 556, 134 (2006).

[37] S. Agostinelli et al. (GEANT4 Collaboration), Nucl. Instrum. Methods Phys. Res., Sect. A 506, 250 (2003).

[38] A. Drescher, B. Grawe, B. Hahn, B. Ingelbach, U. Matthiesen, H. Scheck, J. Spengler, and D. Wegener, Nucl. Instrum. Methods Phys. Res., Sect. A 237, 464 (1985).

[39] G. C. Fox and S. Wolfram, Phys. Lett. 41, 1581 (1978).

[40] B. Aubert et al. (BABAR Collaboration), Phys. Rev. D 66, 032003 (2002).

[41] M.J. Oreglia, Ph.D. thesis, Stanford University [Report No. SLAC-236, 1980], Appendix D.

[42] J.E. Gaiser, Ph.D. thesis, Stanford University [Report No. SLAC-255, 1982], Appendix F.

[43] T. Skwarnicki, Ph.D. thesis, Institute for Nuclear Physics, Krakow [Report No. DESY F31-86-02, 1986], Appendix E.

[44] H. Albrecht et al. (ARGUS Collaboration), Phys. Lett. B
241, 278 (1990).

[45] B. Aubert et al. (BABAR Collaboration), Phys. Rev. D 72, 072003 (2005).

[46] A. Garmash et al. (Belle Collaboration), Phys. Rev. Lett. 96, 251803 (2006).

[47] B. Aubert et al. (BABAR Collaboration), Phys. Rev. D 75, 091103 (2007).

[48] J. Zhang et al. (Belle Collaboration), Phys. Rev. Lett. 94, 031801 (2005).

[49] C. P. Jessop et al. (CLEO Collaboration), Phys. Rev. Lett. 85, 2881 (2000).

[50] B. Aubert et al. (BABAR Collaboration), Phys. Rev. Lett. 91, 201802 (2003).

[51] B. Aubert et al. (BABAR Collaboration), Phys. Rev. Lett. 97, 051802 (2006).

[52] B. Aubert et al. (BABAR Collaboration), Phys. Rev. D 74, 031104 (2006).

[53] O. Long, M. Baak, R. N. Cahn, and D. Kirkby, Phys. Rev. D 68, 034010 (2003).

[54] See, for example, p. 73 of Ref. [14].

[55] M. Gronau and J. Zupan, Phys. Rev. D 71, 074017 (2005).

[56] S. Gardner, arXiv:hep-ph/9906269.

[57] S. Gardner, Phys. Rev. D 72, 034015 (2005).

[58] F. J. Botella, D. London, and J. P. Silva, Phys. Rev. D 73, 071501 (2006).

[59] A. F. Falk, Z. Ligeti, Y. Nir, and H. Quinn, Phys. Rev. D 69, 011502 (2004).

[60] G. Feldman and R. Cousins, Phys. Rev. D 57, 3873 (1998).

[61] M. Beneke, J. Rohrer, and D. Yang, Nucl. Phys. B774, 64 (2007).

[62] B. Aubert et al. (BABAR Collaboration), Phys. Rev. Lett. 91, 171802 (2003).

[63] B. Aubert et al. (BABAR Collaboration), Phys. Rev. Lett. 94, 131801 (2005).

[64] M. Beneke, G. Buchalla, M. Neubert, and C. T. Sachrajda, Phys. Rev. Lett. 83, 1914 (1999).

[65] M. Beneke, G. Buchalla, M. Neubert, and C. T. Sachrajda, Nucl. Phys. B591, 313 (2000).

[66] M. Beneke, G. Buchalla, M. Neubert, and C. T. Sachrajda, Nucl. Phys. B606, 245 (2001).

[67] B. Aubert et al. (BABAR Collaboration), Phys. Rev. Lett. 98, 181803 (2007). 